\title{
Trashcano: Developing a quantitative teaching tool to understand ballistics accelerated by explosive volcanic eruptions
}

\author{
Fabian B. Wadsworth ${ }^{\star \alpha}$, Holly E. Unwin ${ }^{\beta}$, Jérémie Vasseur $\gamma$, \\ Ben M. Kennedy ${ }^{\delta}$, Julia Holzmueller $\gamma$, Bettina Scheu $\gamma$, Taylor Witcher ${ }^{\gamma}$, Janina Adolf $\gamma$, \\ Francisco Cáceres $\gamma$, Ana S. Casas $\gamma$, Valeria Cigala $\gamma$, Alexandra M. Clement ${ }^{\varepsilon}$, \\ Mathieu Colombier $\gamma$, Shane Cronin ${ }^{\zeta}$, Marcel Cronin ${ }^{\eta}$, Donald B. Dingwell $\gamma$, \\ Leticia Freitas Guimarães $\gamma, \theta$, Laura Höltgen $\gamma$, Ulrich Kueppers $\gamma$, Gilles Seropian ${ }^{\delta}$, \\ Sönke Stern $\gamma$, Adrien Teissier ${ }^{\delta}$, Caron E. J. Vossen $\gamma$, Natalie Weichselgartner $\gamma$ \\ ${ }^{\alpha}$ Department of Earth Sciences, Durham University, Durham, DH1 3LE, United Kingdom \\ $\beta$ Department of Earth Sciences, University of Oxford, South Parks Road, Oxford, OX1 3AN, United Kingdom \\ $\gamma_{\text {Earth }}$ and Environmental Sciences, Ludwig-Maximilians-Universiät, Theresienstr. 41, 80333 Munich, Germany \\ ${ }^{\delta}$ Geological Sciences, University of Canterbury, Private Bag 4800, Christchurch 8140, New Zealand \\ ${ }^{\varepsilon}$ Elly und Stoffl, Röngenstrasse 15, 81679 Munich, Germany \\ $\zeta$ School of Environment, University of Auckland, 3A Symonds St, Auckland 1010, New Zealand \\ $\eta$ Bruguera Academy, Carrer de les Orenetes, 08690 santa Coloma de Cervelló, Barcelona, Spain \\ ${ }^{\theta}$ Instituto de Geociências, Universidade de São Paulo. Rua do Lago, 562, Cidade Universitária, \\ São Paulo, SP CEP: 05508-080, Brazil
}

\begin{abstract}
Accurate predictions of volcanological phenomena, such as the trajectory of blocks accelerated by volcanic explosions, require quantitative skills training. Large outdoor experiments can be useful to convey concepts of volcanic processes to students in an exciting way. Beyond the fun aspects, these experiments provide an opportunity to engage with the physics of projectile flight and help promote mathematical learning within the Earth Sciences. We present a quantitative framework required to interpret ballistic trajectories and the outdoor experiment known commonly as "trashcano", taking a step-by-step approach to the physics of this problem, and deriving a range of mathematical solutions involving different levels of complexity. Our solutions are consistent with the predictions from established computer programs for volcanic ballistic trajectory modelling, but we additionally provide a nested set of simplified solutions, useful for a range of teaching scenarios as well as downloadable simulated datasets for use where the full experiment may not be possible.
\end{abstract}

Keywords: Geoscience education; Vulcanian eruption; Calculus; Science outreach

\section{INTRODUCTION}

Teachers, instructors, and lecturers in geoscience at all levels face a problem: that geoscience graduates are not necessarily meeting the needs of an academic or industry workforce in terms of quantitative skill sets [Loudin 2004; Macdonald et al. 2000; Manduca et al. 2008; Wenner et al. 2009; Wenner et al. 2011]. This problem has the associated effect that many incoming graduate students see the geosciences as a more tractable and less-quantitative study choice compared with other natural sciences [Manduca et al. 2008; Wenner et al. 2009]. Conversely, a positive aspect of this problem, is that undergraduate geoscience courses can offer a mechanism for students with less developed physical science backgrounds to break through some perceived hurdles

${ }^{*}$ Corresponding author: fabian.b.wadsworth@durham.ac.uk of "math" and develop quantitative skills necessary for their future work [Macdonald et al. 2000]. In this way the geosciences may be a mechanism for understanding physical science in the same way that the ecological or social sciences often lead into the statistical branch of mathematics. To this end, we aim to provide an example suite of exercises that help students from high school (secondary education), up to undergraduate and postgraduate levels, build quantitative skills applied to a specific problem in the geosciences.

Volcanology is a challenging geoscience topic to teach at any level, from high school (secondary education) to post graduate levels in a university. The modern subject is methodologically broad, incorporating aspects of the natural and social sciences (see Sigurdsson et al. [2015] for an overview of the breadth of subdisciplines in volcanology). Even when concentrating 
on physical or thermodynamic problems within volcanology, such as the dynamics of lava flow emplacement or large eruption plumes, going beyond superficial explanations brings in a raft of physical and chemical complexities. Here, we address one such problem: the trajectory taken by a ballistic volcanic block accelerated from an eruptive vent by an explosion. The understanding of the trajectories of ballistics propelled by an explosion at a volcanic vent is crucial to our prediction of the distribution of hazards around active volcanoes [e.g. Breard et al. 2014; Fitzgerald et al. 2014]. Indeed, the 63 fatalities caused by the September 2014 eruption of Ontake volcano in Japan [Tsunematsu et al. 2016], highlight how hazardous ballistics can be. Ballistic analysis of projectile trajectories can also be key to extracting important information about the source parameters of the initial explosion, such as the energies, subsurface geometries, or explosion mechanisms involved [Blackburn et al. 1976; Chouet et al. 1974; Fagents and Wilson 1993].

In its essence, predicting the flight path of a volcanic block is cognate with the problem of predicting the trajectory of any other projectile in flight; a topic familiar to students of classical mechanics and calculus. There exist useful computer programs to solve this problem at the scales of volcanic environments, such as Eject! [Mastin 2001], which could be used as teaching tools. However, these do not necessitate proficiency with, or understanding of, the underpinning mathematics of the problem, divorcing numerical skills from the output results. To tackle this, we develop a framework in which the full solution to the problem is almost identical in its physical aspects to that given by Eject! [Mastin 2001], but which has nested simplifications, developed step-by-step. In doing so we provide solutions with different levels of predictive accuracy, which are suitable to different levels of mathematical ability. In their entirety, the nested approach developed here demonstrates the mathematical steps an instructor or student can take from an initially complex problem, to various examples of simpler, more manageable problems, and how to assess their respective validity.

Additional challenges facing teachers include the fact that large scale natural phenomena, including volcanic eruptions, are not easily adapted to in-classroom experiments or demonstrations. While volcanic phenomena are pedagogically explicable from a purely theoretical perspective [e.g. Martin 1993], successful learning is thought to be increased when hands-on experiments, non-traditional techniques, or active learning methods are deployed [Beichner et al. 2007; Deslauriers et al. 2011; Freeman et al. 2014; Froyd 2007; Froyd 2008; Haak et al. 2011; Hake 1998; Knight and Wood 2005; Prince 2004]. To incorporate an aspect of active learning, we turn to an experiment commonly called "trashcano" (or sometimes called "bin-bang"; J. Barclay, pers. comm.), in which an explosion is caused by the rapid expansion of over-pressured gas, and drives the acceleration of particles such as ping pong balls (first described by Harpp et al. [2005]). This can be used as a simple demonstration of the phenomenon of projectile motions, or as a rigorous way to compare observations with mathematical predictions, just as volcanologists aim to do during explosive eruptions. By using trashcano, we simplify the problem of projectile trajectories, stripping away many of the complexities associated with real volcanic scenarios, but while keeping the relevant scales and physics and exploring the challenges associated with visual monitoring of projectiles.

Some examples of successful and widely used, scaled in-class experiments or exercises for understanding volcanic systems include fudge factors to explore lava flow rheology [Rust et al. 2008], the M\&M ${ }^{\circledR}$ magma chamber for Bowen crystallization sequences [Wirth 2003], the volcanic hazards simulation for training in crisis communication [Dohaney et al. 2015; Harpp and Sweeney 2002], and the example here, trashcano, for learning about explosive eruption dynamics [Harpp et al. 2005]. Rust et al. [2008] found that using such practical exercises facilitated discussion and improved comprehension of volcanological physical concepts, and better performance in early education within physics or biology has also been found with practical hands-on activities [Penner et al. 1998]. Indeed, many undergraduate volcanology programs involve fun and often largescale class activities, not only to inspire and motivate students but as a serious pedagogic strategy to improve engagement and learning. While we do not undertake an evidence-based approach to demonstrate the pedagogic efficacy of our framework, we take the evidence for the success of active learning techniques as a motivating starting point for designing our study.

In this paper, we describe how to perform the trashcano experiment, how to easily collect and process data from video observations of the explosion, and a mathematical and numerical framework to interpret the results. An illustrative analysis is provided as a guide for the reader, but also to demonstrate the efficacy of this exercise. By providing added quantitative value to a widely used experiment, we also highlight the fit of physics and mathematics to a modern volcanologist's toolbox and promote core STEM (science, technology, engineering, mathematics) skills that are the cornerstone of education policy in many countries around the world.

\section{USING Trashcano fOR TEACHING IN FOUR LEVELS}

Given the range of academic backgrounds encompassed by the average undergraduate or postgraduate cohort in the geosciences in general [Manduca et al. 2008], and volcanology in particular, we provide material developed to satisfy teaching needs in a range of possible teaching scenarios. In Table 1, we break this 
study into 4 levels, each of which has a different suggested teaching level in mind. This table refers to equations throughout this paper (see, for example, section 4) and should be used as a reference table. The levels are not designed to be prescriptive and we leave an assessment of the efficacy of the communication of learning goals in the suggested educational settings to future work.

- Level 1: outreach demonstrations to a public audience or introductory demonstrations at any level.

Using the trashcano experiment described in section 3, without mathematical analysis, may be useful as a simple demonstration of the phenomenon of ballistics accelerated by explosions, providing a starting point for discussions of the mechanics involved. However, even in this simplest level, we propose that discussions of the factors affecting the heights or maximum range of the ballistic particles would enrich the experience for an audience or classroom.

- Level 2: high school (secondary education) or introductory undergraduate students.

We develop a theoretical treatment of projectile trajectories for the case when the initial velocity and launch angle are known (section 4). From this analysis, we arrive at the simple mathematical description of a projectile trajectory in the limiting case where drag is negligible. In combination with the demonstration of the phenomenon using the trashcano experiment (level 1), these solutions may be an effective route to discussion of the mechanics of volcanic ballistics and can provide order-ofmagnitude estimates of the range and height of volcanic blocks. For this simple case, we find the result for the maximum range and height of a projectile.

- Level 3: undergraduate or postgraduate students.

If we make a simplifying assumption about the way drag acts on projectiles, we can additionally account for drag forces and arrive at an analytical solution that can be used to quantitatively predict the trajectory of both the projectiles from trashcano, and those from volcanic eruptions. This requires manipulation of equations, and knowledge of calculus techniques for integration would be advantageous.

- Level 4: undergraduate or postgraduate students.

The full solution is presented, for which a numerical solution is required. We provide an example method to solve the governing equations using Python $^{\mathrm{TM}}$, which may require some background in computational methods or could be incorporated into courses specifically designed to teach computational methods.
The experiment itself can be performed in under 1 hour, including introductory discussion and a safety briefing, making it ideal for short exercises at level 1. For level 2, we anticipate that a follow-up 20 minute data-collection exercise should focus on estimating the maximum, minimum, and mean range of each particle type ejected from the explosion. A 1-2 hour laboratory practical will be essential, in which the simple calculations required to predict height and range of ballistics are introduced, and those predictions are compared with the empirical data.

At levels 3 or 4 , there are different ways this exercise could be delivered, depending on the context of the course in which it is deployed, each of which would require different time allocation and preparation. At its most basic, levels 3 and 4 would require a first preparatory laboratory exercise (1-2 hours) to follow the derivations given in section 4 . At these levels, the data collection is more involved than at level 2, because individual particles must be tracked using video. For this reason, the exercise itself is likely to last up to an hour. A follow-up laboratory practical after the experiment will be required to process and analyze the data collected during the experiment (1-2 hours). The instructor can reduce the time required for this second practical by having the students prepare a method in advance (using either Python ${ }^{\mathrm{TM}}$, Microsoft ${ }^{\circledR}$ Excel, or other data manipulation tools) to compare the empirical data from processed video, with the predictions discussed in the first preparatory practical. We note that level 4 requires proficiency on the part of the instructor with simple computational methods, and the use of the freely available Python ${ }^{\mathrm{TM}}$ platform, or equivalent, to find numerical solutions to coupled equations. If the instructor does not have the necessary experience with computational methods, then an alternative for Level 4 , is for the instructor to use the computer program Eject! [Mastin 2001], which is discussed later.

\section{THE EXPERIMENT}

Following the classic examples of the trashcano setup [Harpp et al. 2005], we used a large, 60-80 litre bucket as the experimental volcanic vent analogue. A 1.5-litre soft-drink bottle was filled to approximately half-way with liquid nitrogen using a funnel, sealed, and placed in the bottom of the bucket. Relevant safety precautions were taken, including the use of protective clothing and shielding for handling liquid nitrogen. We did not fill the bucket with water in the experiment, as suggested in some descriptions of trashcano, so that the projectile velocities were easier to track upon explosion. This also negates the need for the bricks around the explosive charge (step 3 in the description from Harpp et al. [2005]). Without the damping effect of water in the bucket, the explosion can be particularly energetic, and often ruptures the bucket wall violently. For this rea- 
Table 1 - Example of compartmentalized uses of this study.

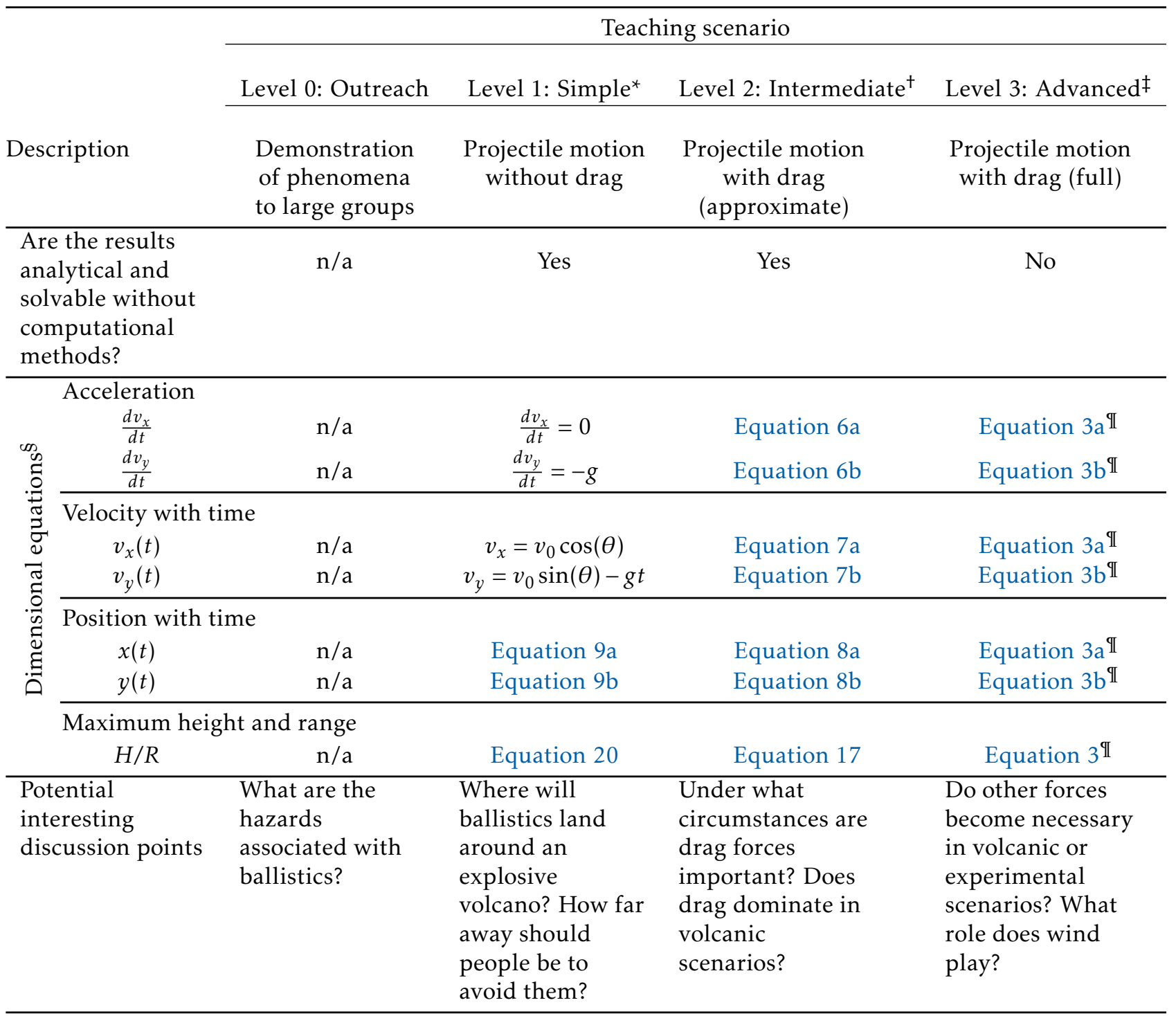

* e.g. various secondary education levels.

t e.g. undergraduate or postgraduate.

$\ddagger$ e.g. undergraduate or postgraduate

$\S$ Here we state where to find the dimensional solutions and the reader is referred to the main text for the dimensionless equivalents.

II Numerical solution: refer to Listing 1.

son, we advise caution, and the use of a sturdy, thickwalled bucket. Figure 1 shows a typical trashcano example from the Ludwig-Maximilians-Universität.

Onto the bottle of liquid nitrogen, we poured experimental projectiles. In our case we chose 2350 pingpong balls, $\sim 50$ indoor foam balls, $\sim 170$ soft bouncy balls, and 1 mini-football/basketball (all pictured in Figure 2; we recommend using projectiles with strong colours to facilitate easy particle tracking). The time between the sealing of the plastic bottle and the explosion can vary, dominantly depending on the filling fraction of nitrogen, the bottle volume, the type of plastic from which the bottle is manufactured, and the thickness of the bottle plastic. Caution is advised as the time between sealing the bottle and the explosion can be as short as $\sim 10$ seconds*, but can be as long as 15 minutes. Once the bottle is sealed and the experiment is started, no one should touch the bottle. The explosion is loud and ear protection as well as other safety clothing or equipment is recommended if observers are standing

\footnotetext{
${ }^{*}$ See this video example from the "Magical Magma" lecture by J. Barclay and R. Herd; https://www.youtube.com/watch?v=NFp4hbaBkYU (go to time 51:15 for the trashcano demonstration).
} 

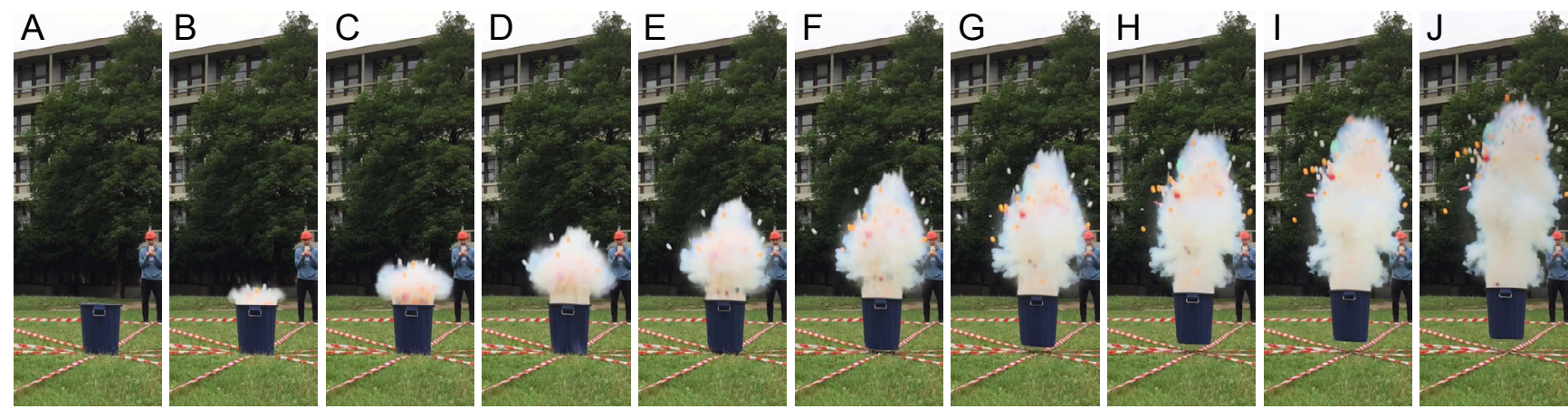

Figure 1: One example of the trashcano experiment as an equally-spaced time-series (from left to right) of photographs showing the evolution of the projectile trajectories.

relatively close by (within approximately $5 \mathrm{~m}$ ). We marked a grid on the grass in radial coordinates around the bucket vent so that the students could measure the distribution of particles after the experiment ended.

We divided the student workforce into a "monitoring team" responsible for video acquisition of the particle trajectories, a "sedimentology team" or "field volcanology team" responsible for investigating the distribution of ejecta after deposition, and a "hazards team" responsible for safety of fellow students and bystanders.

To make this practical experiment easy to perform and appealing to students who identify with their own devices, we used hand-held smartphone cameras as the video recording tool. The "monitoring team" were armed with phone cameras and placed at various positions (not more than $15 \mathrm{~m}$ distance) around the impending explosion and recorded continuously from the moment the bottle was placed in the bucket. The resulting videos were downloaded and the frame rate and time-stamps were recorded as well as the distance of the observer from the vent, termed $D$. Using handheld cameras requires post-experiment image processing techniques to stabilize the images (described below) and so it can be easier to use cameras fixed on tripods, if available.

In our experiments, the dominant quantifiable uncertainty comes from camera spatial resolution and camera frame rate. In our case, the two videos used to process data were at the same distance from the vent and used the same camera type and settings, and therefore these uncertainties could be quantified as resulting in $\pm 0.05 \mathrm{~m}$ and $\pm 0.03 \mathrm{~s}$ on all lengths and times, respectively. The uncertainty on length and time are maxima and are derived from the frame resolution compared with the scale of known length, and frame rate of normal-speed videos captured with typical phone cameras at the time of writing. From these uncertainties, we can propagate uncertainties on velocities.

After the explosion, the "sedimentology team" recorded the distance of the projectiles from the vent. It is useful for this reason to perform the experiment on grass because the projectiles do not bounce significantly when they land.
If video stabilization is required to correct movement of the hand-held cameras during filming, a variety of open-source software can be used. The majority of the videos of the experiment were stabilized using the motion tracking feature of Blender ${ }^{\dagger}$. The motion tracking feature can follow points in the video as the camera moves. The program then inverts the apparent scene motion in the video, to recover the varying camera orientation and produce a stabilized final product. For tracking to be successful there must be sufficient suitable points for the software to track. Placing a number of brightly colored objects around the area in which the experiment is to take place will help the software to successfully track points through camera shake. Stabilization using the motion tracking feature of Blender is generally unsuccessful for videos taken at close range, because the particles flying through the video hide the points being tracked. These videos can be stabilized using Deshaker [Thalin 2013], a plugin for VirtualDub. While Blender is potentially easier to use, Deshaker determines the movement between each frame and attempts to remove it, such that the software can manage to stabilize those videos where Blender cannot follow enough tracking points. Once the videos were stabilized, the particles were tracked using MtrackJ [Meijering et al. 2012], a plugin for ImageJ [e.g. Schneider et al. 2012], which can be used to manually find the projectile(s) in each frame and record them as an apparent vertical and horizontal position $y(t)$ and $x(t)$ in the images. We use the "vent" width in the images as the scale bar. We note that using a single camera position, these apparent vertical and horizontal positions are not the real position of any given projectile. Some aspects of the relationship between observer and projectile, including distortion, 3-dimensional tracking with multiple cameras, and corrections will be discussed later.

A correction to the $x$ - and $y$-positions in the images must be made if the projectiles reach large maximum horizontal distances or heights relative to the observer, respectively. This correction adjusts for any apparent difference between the calculated position in an image and the actual position in reality, of a projectile asso-

\footnotetext{
${ }^{\dagger}$ Available from https://www.blender.org/
} 
A

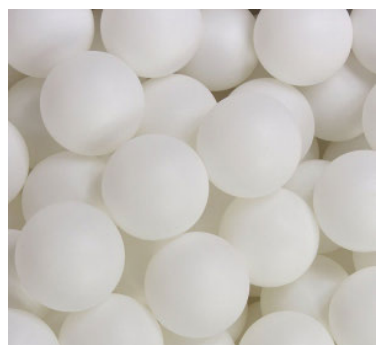

B

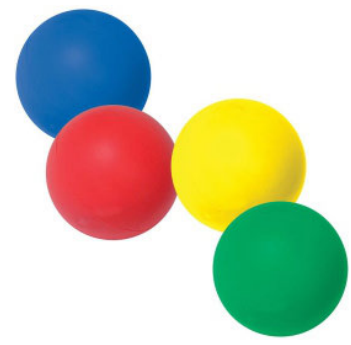

C

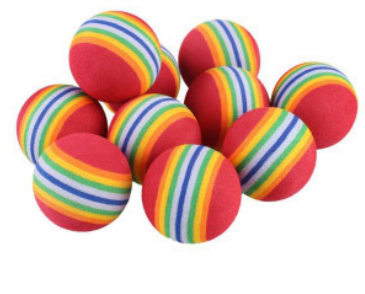

D

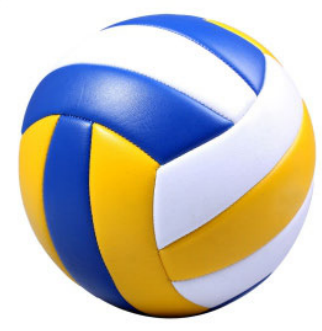

Figure 2: Images of the experimental projectiles used here. [A] Ping-pong balls with radius $a=0.014 \mathrm{~m}$ and bulk density $\rho_{p}=80 \mathrm{~kg} \mathrm{~m}^{-3}$. [B] Foam indoor balls with $a=0.035 \mathrm{~m}$ and $\rho_{p}=100 \mathrm{~kg} \mathrm{~m}^{-3}$. [C] Bouncy balls with $a=0.02 \mathrm{~m}$ and $\rho_{p}=94 \mathrm{~kg} \mathrm{~m}^{-3}$. [D] A mini-football with $a=0.06 \mathrm{~m}$ and $\rho_{p}=90 \mathrm{~kg} \mathrm{~m}^{-3}$. We note that colored ping-pong balls are easier to track in videos.

ciated with the large distances from an observer, and is $x=D \tan \left(\sin ^{-1}\left[x^{*} / D\right]\right)$, in the case of the horizontal position data, and $y=D \tan \left(\sin ^{-1}\left[y^{*} / D\right]\right)$, in the case of the vertical position data. Here $x^{*}$ and $y^{*}$ are the apparent recorded horizontal and vertical positions. In detail, we find that we only require the correction to the $y$-positions, as the horizontal motion is much less than the vertical motion, such that the correction to the horizontal positions is within the error of the recorded raw apparent position data. As well as recording apparent positions, MtrackJ measures the angle $\theta$ relative to an initial point and a given line (we chose vertical).

\section{TheORETICAL BACKGROUND AND MATHEMAT- ICAL FRAMEWORK: POINT-SOURCE BALLISTIC TRAJECTORIES FROM A KNOWN INITIAL VE- LOCITY AND LAUNCH ANGLE}

This theoretical section is designed to lay out the solutions around the volcanic ballistics explosion problem, for either a straightforward natural example, or the outdoor laboratory exercise. We consider a particular case of a projectile that is subject to drag and gravitational forces only and which is moving through a stagnant fluid phase (air in our case). Other forces that may be relevant in the natural case, as well as the effects of the fluid (air) velocity, are discussed later.

We develop our solution to the problem of predicting a projectile's trajectory in three pedagogic stages: (1) we develop the equations of motion, (2) we provide an analytical approximation for the equations of motion and assess the validity of this, (3) we provide limiting simple cases. Finally we non-dimensionalize the problem to provide results that can be compared with data at any scale.

\subsection{The full solution}

When an object is in motion, such as a projectile tracking its course, the sum of forces opposing motion must balance the force driving motion. If the mass $m$ of a projectile is constant, from Newton's second law, we can assume the force arising from its acceleration is $m d \mathbf{v} / d t$ where $\mathbf{v}$ is the velocity of the motion and $t$ is time. In all cases of practical interest, the forces opposing motion are the drag force $\mathbf{F}$ and the force due to gravity $\mathbf{F}_{\mathbf{g}}=m \mathbf{g}$ where $\mathbf{g}$ is the local acceleration due to gravity. By balancing these components, we have:

$$
m \frac{d \mathbf{v}}{d t}=\mathbf{F}+m \mathbf{g}
$$

If we assume the density and viscosity of the fluid through which the projectile is moving are negligible, then the drag force $\mathbf{F}$ can be parameterized as

$$
\mathbf{F}=\frac{1}{2} \rho A C v \mathbf{v}
$$

where $\rho$ is the density of the fluid (air in this case), $A$ is the cross-sectional area exposed to the flow streamlines, $C$ is the coefficient of drag, and $v$ is the norm of the vector $\mathbf{v}$ (see below). Throughout most of what follows, we assume that $C$ is a constant, consistent with drag in the "intermediate" regime of Reynolds number [Clift et al. 2005]. In reality, $C$ is a function of the particle Reynolds number where that dependence is especially important at low Reynolds number-the Stokes regime-and high Reynolds number above a critical transition (see Clift et al. [2005] for a compilation). Later, we will compare our results to those derived when $C$ is computed continuously as a function of Reynolds number [Mastin 2001].

If we consider that the trajectory of our projectile remains in a 2D Cartesian plane, then the in-plane directions $x$ (horizontal) and $y$ (vertical) can define the coordinate system for Equations 1 and 2, which, assuming the force $m \mathbf{g}$ only acts in the $y$-direction, gives

$$
\begin{aligned}
& \frac{d v_{x}}{d t}=-\frac{F_{x}}{m}=-\frac{\rho A C}{2 m} v v_{x} \\
& \frac{d v_{y}}{d t}=-\frac{F_{y}}{m}-g=-\frac{\rho A C}{2 m} v v_{y}-g
\end{aligned}
$$


where $v_{x}$ and $v_{y}$ are the horizontal and vertical components of the projectile velocity. Similarly, $F_{x}$ and $F_{y}$ are the drag force components in the horizontal and vertical directions, respectively. In these coordinates, $v=\|\mathbf{v}\|=\left(v_{x}^{2}+v_{y}^{2}\right)^{1 / 2}$.

Equation 3 is not analytically tractable because the amplitude of the drag force, $F$, is proportional to the square of the speed of the projectile, $F \propto v^{2}$. This set of equations can be solved using numerical methods (discussed later). Additional complexities can be incorporated as additive force vectors in Equation 1, which may be relevant in volcanic scenarios, such as the lift or Magnus force [de'Michieli Vitturi et al. 2010; Taddeucci et al. 2017], which are neglected here and which have different dependencies on $v$ but are discussed later.

\subsection{An approximate solution}

As stated, the problem with finding a solution to Equations $3 \mathrm{a}$ and $3 \mathrm{~b}$ arises from the form of $\mathbf{F}$ given in Equation 2. To solve this more easily, we can replace Equation 2 with a form in which $\mathbf{F}$ is linearly dependent on $v$, such as

$$
\mathbf{F}=B \mathbf{v}
$$

where $B$ is a constant with dimensions of mass per unit time (a mass flux). Doing this has the implication that $F \propto v$, and in turn, this implies that the additional velocity that appears in Equation 2 is now set to be constant and included in $B$. The validity of this approximation will be assessed later.

Combining Equation 4 with Equation 1 and projecting onto our coordinate system yields

$$
\begin{aligned}
& \frac{d v_{x}}{d t}=-\frac{F_{x}}{m}=-\frac{B}{m} v_{x} \\
& \frac{d v_{y}}{d t}=-\frac{F_{y}}{m}-g=-\frac{B}{m} v_{y}-g .
\end{aligned}
$$

Another conceptual tool that will become useful in our understanding of the problem is to define a terminal velocity scale that is $v_{t}=m g / B$. Then Equation 5 becomes

$$
\begin{aligned}
& \frac{d v_{x}}{d t}=-g \frac{v_{x}}{v_{t}} \\
& \frac{d v_{y}}{d t}=-g\left(\frac{v_{y}}{v_{t}}+1\right) .
\end{aligned}
$$

Defining an initial launch velocity of $v_{0}$ and an initial launch angle $\theta$, such that $v_{x, 0}=v_{0} \cos (\theta)$ and $v_{y, 0}=v_{0} \sin (\theta)$, we can integrate Equation 6 from $t=0$ to $t$ and find that

$$
\begin{aligned}
& v_{x}=v_{0} \cos (\theta) \exp \left(-\frac{g}{v_{t}} t\right) \\
& v_{y}=v_{0} \sin (\theta) \exp \left(-\frac{g}{v_{t}} t\right)-v_{t}\left[1-\exp \left(-\frac{g}{v_{t}} t\right)\right] .
\end{aligned}
$$

If we integrate a second time to convert $v_{x}(t)$ and $v_{y}(t)$ to the two components of the position $x(t)$ and $y(t)$, we get

$$
\begin{aligned}
& x=v_{0} \cos (\theta) \frac{v_{t}}{g}\left[1-\exp \left(-\frac{g}{v_{t}} t\right)\right] \\
& y=\left(v_{0} \sin (\theta)+v_{t}\right) \frac{v_{t}}{g}\left[1-\exp \left(-\frac{g}{v_{t}} t\right)\right]-v_{t} t .
\end{aligned}
$$

such that Equation 8 would allow us to predict the trajectory of a projectile as a function of time, knowing $v_{0}$ and $\theta$ and having empirical constraint of $v_{t}$.

\subsection{Useful limiting behaviour of the approximate so- lution}

There are limits in which the behaviour of a projectile will be simpler than predicted by Equation 8 . The first is when $t \ll v_{t} / g$, which is when $d v_{x} / d t=0$ and $d v_{y} / d t=-g$, leading to the solutions in which $\mathbf{F}$ is negligible (zero drag in a vacuum):

$$
\begin{aligned}
& x=v_{0} \cos (\theta) t \\
& y=v_{0} \sin (\theta) t-\frac{1}{2} g t^{2} .
\end{aligned}
$$

which can also be derived from first principles via Equation 1 by setting $\mathbf{F}=0$. If drag were negligible throughout the flight, Equation 9 defines the classic parabola of a projectile in the simplest case.

In the opposite limit when $t \gg v_{t} / g$, then

$$
\begin{aligned}
& x=\frac{v_{t}}{g} v_{0} \cos (\theta) \\
& y=\frac{v_{t}}{g}\left[v_{0} \sin (\theta)+v_{t}\right]-v_{t} t .
\end{aligned}
$$

which has the desirable limiting property that $v_{x}=0$ and $v_{y}=-v_{t}$, such that in the limit of long times and when drag is important, the projectile falls vertically downward at terminal velocity.

\subsection{Dimensionless solutions}

Dimensional analysis is a powerful tool for scaling natural phenomena and predicting expected behaviour, 
particularly to a first-order approximation. In our problem, a useful substitution would be to define the characteristic timescale of the projectile $\lambda=v_{0} / g$ and a characteristic length scale of the flight as $L=v_{0}^{2} / g$. This leads to a dimensionless time as $\bar{t}=t / \lambda$ and a dimensionless $x$ - and $y$-coordinate scale as $\bar{x}=x / L$ and $\bar{y}=y / L$. These scales can be used to nondimensionalize the governing equations presented in the previous sections.

We also introduce dimensionless control parameters $K_{1}$ and $K_{2}$, which will contain all of the materialproperty constants in our problem for the full and approximate solution, respectively. These are

$$
K_{1}=\frac{2 m g}{\rho A C v_{0}^{2}} \text { and } K_{2}=\frac{v_{t}}{v_{0}}=\frac{m g}{B v_{0}} \text {. }
$$

We can see that $K_{1}$ and $K_{2}$ are similar and would be identical if $B=\rho A C v_{0} / 2$ : the definition of our approximation made earlier. The full solution (Equation 3) now becomes

$$
\begin{aligned}
& \frac{d \bar{v}_{x}}{d \bar{t}}=-\frac{1}{K_{1}} \bar{v} \bar{v}_{x} \\
& \frac{d \bar{v}_{y}}{d \bar{t}}=-\frac{1}{K_{1}} \bar{v} \bar{v}_{y}-1,
\end{aligned}
$$

where we use a bar above a parameter to denote it is dimensionless.

To non-dimensionalize the approximate solutions given in Equations 7 and 8, we have that

$$
\begin{aligned}
& \bar{v}_{x}=\cos (\theta) \exp \left(-\frac{\bar{t}}{K_{2}}\right) \\
& \bar{v}_{y}=\sin (\theta) \exp \left(-\frac{\bar{t}}{K_{2}}\right)-K_{2}\left[1-\exp \left(-\frac{\bar{t}}{K_{2}}\right)\right],
\end{aligned}
$$

and that

$$
\begin{aligned}
& \bar{x}=K_{2} \cos (\theta)\left[1-\exp \left(-\frac{\bar{t}}{K_{2}}\right)\right] \\
& \bar{y}=K_{2}\left[\left(\sin (\theta)+K_{2}\right)\left[1-\exp \left(-\frac{\bar{t}}{K_{2}}\right)\right]-\bar{t} .\right.
\end{aligned}
$$

The case of zero drag has the simple dimensionless limiting form $\bar{x}=\cos (\theta) \bar{t}$ and $\bar{y}=\sin (\theta) \bar{t}-\bar{t}^{2} / 2$, and in the case where drag is dominant, $\bar{x}=K_{2} \cos (\theta)$ and $\bar{y}=K_{2}\left[\left(\sin (\theta)+K_{2}\right)-\bar{t}\right]$.

\subsection{Comparison of the full and approximate solutions}

In Figure 3 we provide solutions of the full and approximate models, plotted as the evolution of the two components of velocity, or as the full trajectory to demonstrate how the limiting behaviour is met at small and large times as described above. In Figure 4 we demonstrate how the two models diverge when $K_{1}=K_{2}$, which is useful for assessing the validity of the approximation made earlier. This assessment is cast as the difference between the solutions using a weighted sum of the difference in $\bar{y}$, where the full model is termed $\bar{y}_{f}$ and the approximation is termed $\bar{y}_{a}$, and the weighted sum of the difference is $\sum_{i=1}^{i=N}\left(\bar{y}_{a, i}-\bar{y}_{f, i}\right) / N$, where $N$ is the number of data points at $\bar{y}=0$ for $\bar{t}>0$. Large deviations from zero represent large discrepancies between the two solutions where negative values imply that the approximation of the full model underestimates the trajectory of the projectile compared with the full solution. Of course, in any real situation, $K_{1}$ need not be equal to $K_{2}$ and both could be treated as fit parameters to measured data.

4.6 Solutions for the maximum height and range of a projectile

During a projectile's flight, the maximum height and range (the point at which the projectile lands) can be empirically determined easily. Therefore, it is informative to extract solutions for these positions from the equations presented above. These are especially useful quantities to derive for classroom exercises where the full treatment (section 6) may not be appropriate.

For simplicity, we assume that the launch position and the position at which the projectile lands are at the same $y$, implying flat ground. With this assumption, we can solve the approximate solution for the maximum height $H$ (when $v_{y}=0$ ) and the range $R$ (when $y$ is zero excluding the trivial solution of $y=0$ at $t=0$ )

$$
H=\frac{v_{t}^{2}}{g}(\beta-1-\ln \beta)
$$

$$
R=\frac{v_{t}^{2}}{g} \frac{\beta-1}{\tan (\theta)}(1-\exp [-(W[-\beta \exp (-\beta)]+\beta)])^{-1}
$$

$\left.\frac{H}{R}=\left(1-\frac{\ln \beta}{\beta-1}\right) \tan (\theta)(1-\exp [-W[-\beta \exp (-\beta)]+\beta)]\right)^{-1}$

where $\beta=\sin (\theta) / K_{2}+1$ and $W$ is known as the Lambert $\mathrm{W}$-function (or sometimes known as the omega function). The use of $W$ may render Equations 16 and 17 less easily tractable, and so it is useful to also consider the limiting cases. As before, the limiting case where $t \ll v_{t} / g$ refers to when the drag force is negligible and the height and range become

$$
\begin{aligned}
& H=\frac{v_{0}^{2}}{2 g} \sin ^{2}(\theta) \\
& R=\frac{v_{0}^{2}}{g} \sin (2 \theta)
\end{aligned}
$$



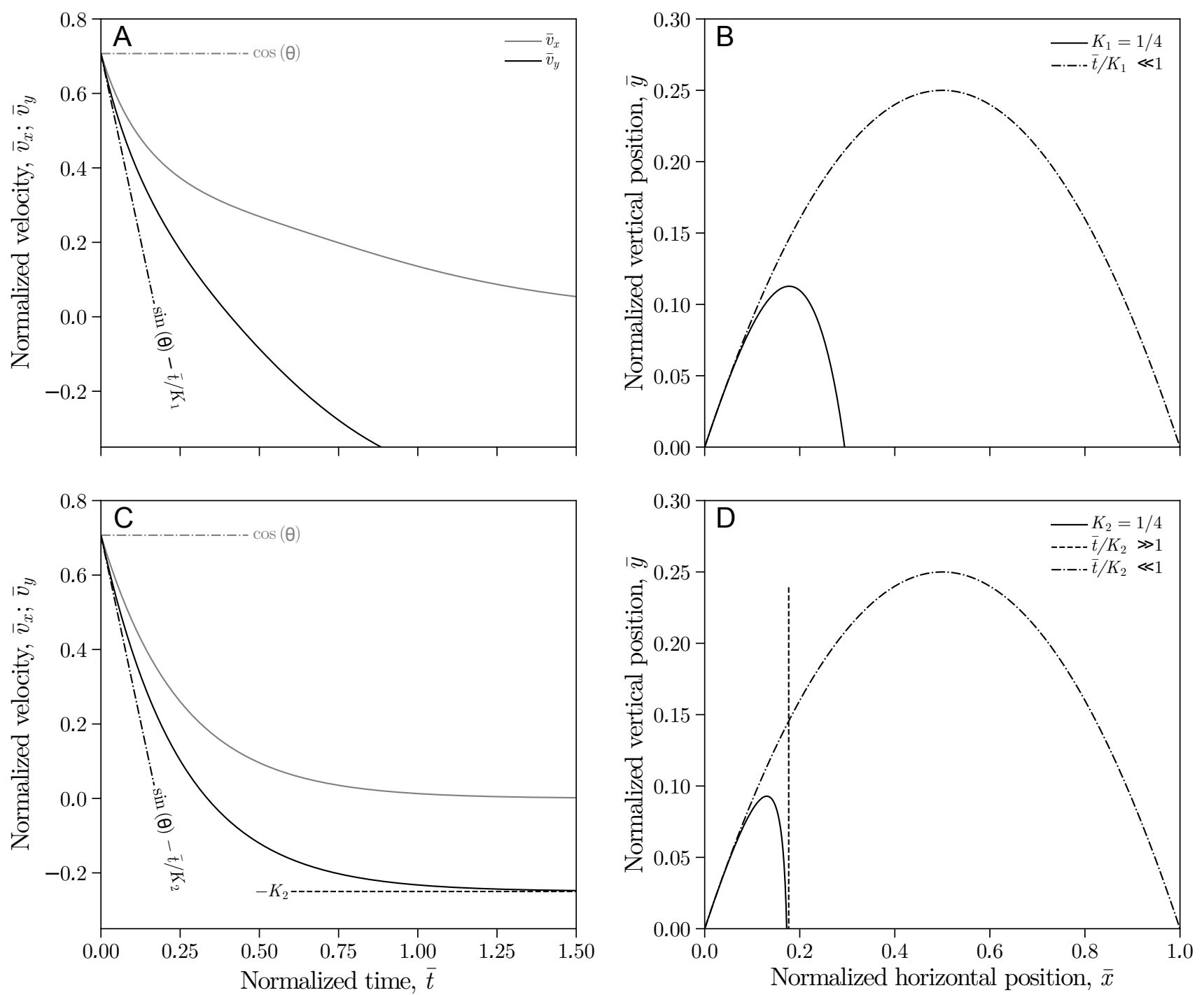

Figure 3: The predictions of the $[\mathrm{A}]-[\mathrm{B}]$ full and $[\mathrm{C}]-[\mathrm{D}]$ approximate model where the approximate model can be compared with limiting solutions for end-member conditions. In both cases, we use $\theta=\pi / 4$ and we use $K_{1}$ and $K_{2}$ of $1 / 4$. The models would be more similar to one another if $K_{1}$ and $K_{2}$ were $>10^{1}$ (see Figure 4 ) but we choose a value of $K_{1}$ and $K_{2}$ that demonstrates how different the models can be.

$$
\frac{H}{R}=\frac{1}{4} \tan (\theta)
$$

And when the other limit is met, such that $t \gg v_{t} / g$ and drag can come to dominate, we have

$$
R=\frac{v_{0} v_{t}}{g} \cos (\theta)
$$

where, in this limit, there is no result for $H$.

4.7 Relating the initial velocity to source parameters of the explosion

Since the well-known example of how G.I. Taylor estimated the source parameters for the nuclear explosive used in the Trinity bomb test carried out in 1945 [Taylor 1950], the utility of dimensional analysis to scale for the energetics of an explosion has been established. In our case, extracting source parameters from the observed behaviour of the explosion, can be rendered somewhat simpler. In the previous section, the equations of motion rely on an initial velocity (and launch angle) in order to find solutions for the trajectory of the projectile. While initial velocities are observables that we can measure, in applying the results of the previous section to real examples of projectile trajectories, the initial velocities do not immediately tell us something about the explosion itself. It is important to make this connection between the distribution of initial velocities of projectiles and the explosion energy because, for problems of volcanic interest, this explosion energy typically relates to the overpressure state of the magma prior to explosion [Linde et al. 2010; Mueller et al. 2008], an important source parameter. 


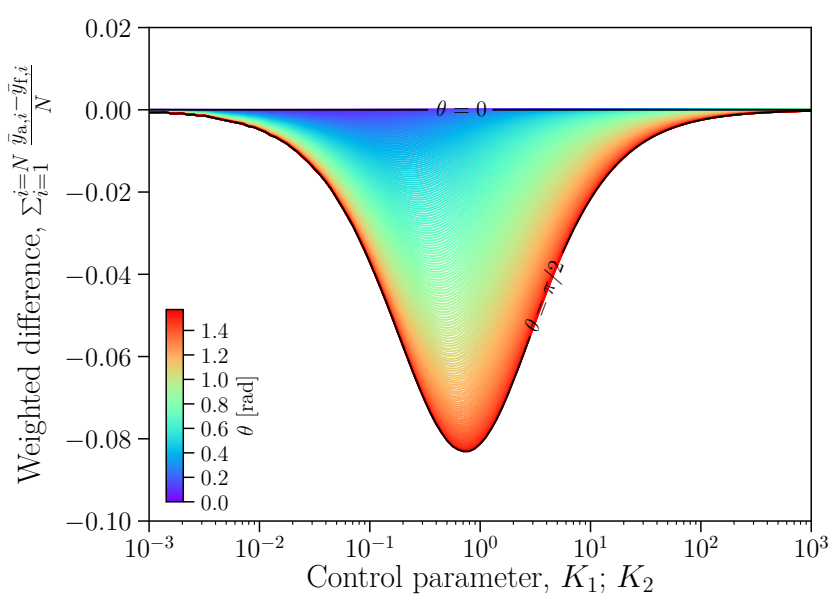

Figure 4: Comparison between the full numerical solution and the approximation where $\theta$ is quoted in radians for convenience. Here the difference between the solutions is cast as the weighted sum of the difference in $\bar{y}$, where the full model is termed $\bar{y}_{f}$ and the approximation is termed $\bar{y}_{a}$, and the weighted sum of the difference is $\sum_{i=1}^{i=N}\left(\bar{y}_{a, i}-\bar{y}_{f, i}\right) / N$. We note that the difference is negligible in the limit of $K_{1} \rightarrow \infty$ (and $K_{2} \rightarrow \infty$ ), corresponding to small $v_{0}$ or negligible drag, and in the limit $K_{1} \rightarrow 0$ (and $K_{2} \rightarrow 0$ ) where drag dominates or $v_{0}$ is very large. The latter limit occurs simply because the projectile motion is small. The maximum discrepancy is at $K_{1} \sim \mathcal{O}(1)\left(K_{2} \sim \mathcal{O}(1)\right)$.

We take the explosion energy to be $E$ and the kinetic energy of each projectile to be $E_{k}$ and $E_{k, 0}=m v_{0}^{2} / 2$ is the initial kinetic energy of each projectile that is an observable parameter. Initially, we make a crude simplifying assumption that the explosion energy should be close to the sum of all the projectile kinetic energies $E \simeq \sum E_{k, 0}$. This is likely to result in an underestimate of $E$ given that in reality there are energetic losses in the sound and heat produced in the explosion and the acceleration of the gas phase. However, finding $\sum E_{k, 0}$ from observable data is tractable and will provide us with a scaling argument for $E$ that can be tested against other estimates of $E$ later.

\section{EXPERIMENTAL RESULTS AND ANALYSIS}

Here we analyze our results as an example of the outputs that can be found using the model and experiment described. Processed data is supplied as a supplementary data file for use in classrooms where the experiment may not be feasible.

5.1 The evolution of the vertical component of velocity

Tracking of $x(t)$ and $y(t)$ can be converted to $v, v_{x}$ and $v_{y}$. We confine our analysis of velocities to $v_{y}(t)$ because there is a large uncertainty on $v_{x}(t)$ due to the unknown 3 -dimensional aspect, which is analyzed in detail later. Although the uncertainty in $v_{x}(t)$ also conveys an uncertainty on $v_{y}(t)$, the latter is deemed much less because the launch angles tend to be high. In Figure 5, we show the evolution of $v_{y}$ for each clast type. In all cases there is a non-linear decay in the vertical component of the velocities that goes through zero when the projectiles reach the apex of their trajectory. The whole process is complete in $\sim 4$ seconds. All datasets for each particle type appear reasonably similar, probably due to the similar density of the particles used. Normalizing the $v_{v}$ data by $v_{0} \sin (\theta)$ allows them to decay from unity. We take a two-step approach to the comparison between the data and the models. First, we use all data together to compare the full and approximate models, and second, we use each projectile-type separately. In both cases, we fit for $v_{0}$ using a least-squares regressive approach (follow Kemmer and Keller [2010] for a user-friendly procedural introduction) because determination of $v_{0}$ from the videos is hampered by the initial phase of nitrogen evaporation that obscures the projectiles. Fitting for $v_{0}$ does not significantly affect the result, compared with empirically determining it. In the case of the full model, we additionally fit for $K_{1}$, and, in the case of the approximate model, we fit for $K_{2}$. When we use all data, we find a global average $\left\langle v_{0}\right\rangle=31.6 \mathrm{~m} \mathrm{~s}^{-1}$ using the full model (Equation 3 or Equation 12), and $\left\langle v_{0}\right\rangle=24.0 \mathrm{~ms}^{-1}$ using the approximate model (Equation 7 or Equation 13; Figure 5A). These values are not significantly different when we analyze the individual projectile types (see Figure 5B-E). In detail, we fit using the dimensional models (Equation 3 and Equation 7) rather than the dimensionless forms, but this does not affect the solution.

The values of $K_{2}$ from the approximate solution are simply used to adjust the model and can't be clearly interpreted. However, the values of $K_{1}$ contain information that can be decomposed using Equation 11. In the relationship $K_{1}=2 m g /\left(\rho A C v_{0}^{2}\right), \rho, A, m$ and $g$ are known quantities. As stated, $v_{0}$ is an independent fit parameter. Therefore, the remaining unknown that can be interpreted from the values of $K_{1}$ is the drag coefficient $C$. We find that all the projectiles have a similar value $0.05 \leq K_{1} \leq 0.38$, which, given the appropriate values of the constants in Equation 11, would relate to $0.35<C<0.48$. All the particles are spheres, and so we can compare these values with the standard drag curve [Clift et al. 2005], which predicts that at intermediate Reynolds numbers, $C \sim 0.5$. Clearly, our fitted values of $C$ are in reasonably good agreement with intermediateReynolds number flow (within a factor of 2). Differences between the fitted $C$ and the value $C=0.5$ may be due to a non-zero gas velocity $u$ that is not given in our model [see Taddeucci et al. 2017]. If the gas velocity is positive (in the same direction as the particle motion), then we would expect the apparent $C$ we compute to be less than the value in stagnant air. We have not incor- 

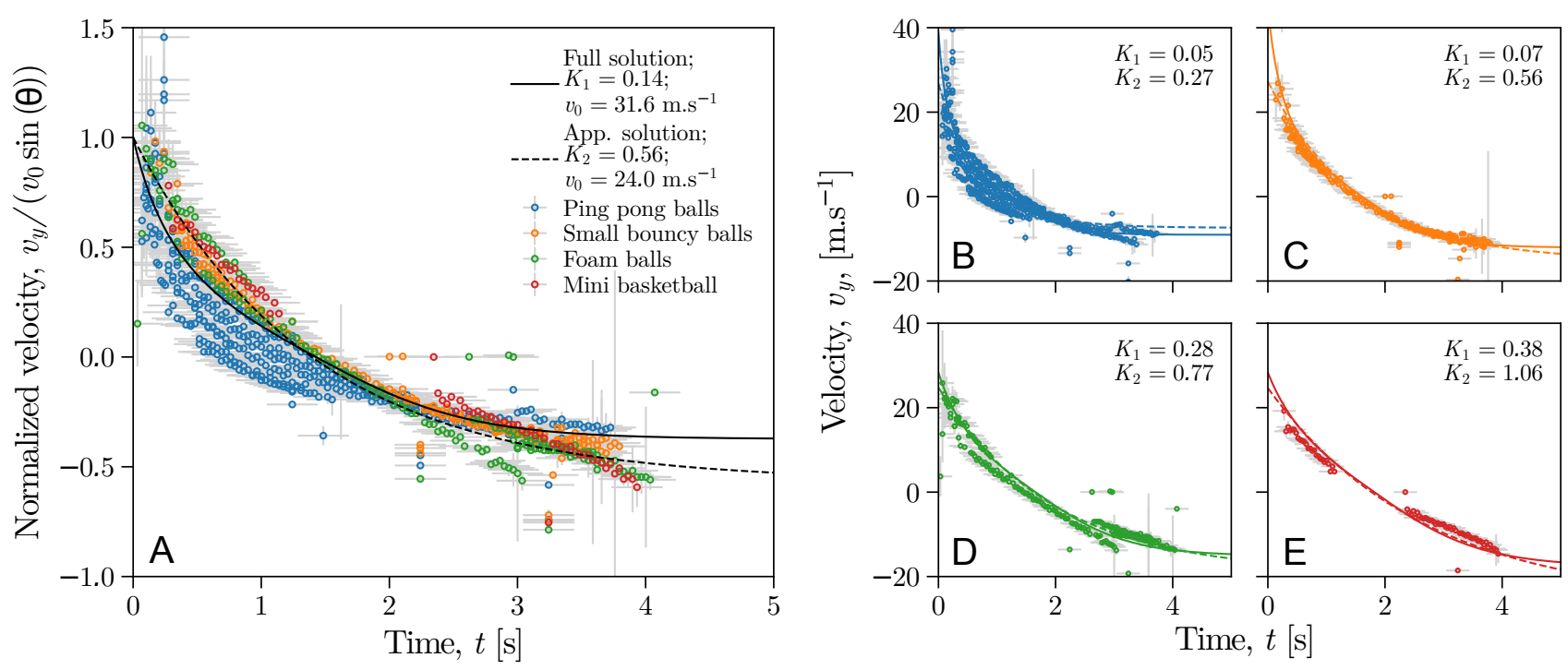

Figure 5: Results for $v_{y}(t)$ for our four types of projectile compared with model results. The fitted parameters used in the model curve results are given in Table 2 and are discussed in the text. Panel [A] describes the data as $v_{y} /\left(v_{0} \sin (\theta)\right)$ for all particles, showing that the evolution can be described similarly in all cases with a global fit. Panels $[\mathrm{B}]-[\mathrm{E}]$ show individual fits for individual particle populations.

porated an account of $u(t)$ in our analysis because it is not easily measurable nor is it easy to predict from first principles. However, by rearranging Equation 11, and incorporating a non-zero initial gas velocity $u_{0}$ we have

$$
C=\frac{2 m g}{\rho A K_{1}\left(v_{0}-u_{0}\right)^{2}} .
$$

We can set $C=0.5$ [Clift et al. 2005] in Equation 22 to find that $0.8 \leq u_{0} \leq 7.5 \mathrm{~m} \mathrm{~s}^{-1}$ for our range of fitted $K_{1}$ given above. This implies that the expanding cloud of nitrogen (see Figure 1) has a small effect on the projectile motion. A similar conclusion of "reduced drag" effects in situations where gas velocities are nonzero and in the direction of motion of the projectiles, was reached when analysing complex eruption ballistic data [Lube et al. 2014] and may be common in a range of explosive blasts [Fagents and Wilson 1993]. However, the particles quickly overtake the cloud of nitrogen and enter the region of air that we assume is close to being stagnant, in the absence of wind.

\subsection{Height and range}

The "sedimentology team" measured the distribution of distances from the source vent for each projectile assuming they did not bounce significantly upon impact with the grass. This is equivalent to the distribution of ranges $R$, which are given as a histogram in Figure 6. The arithmetic mean $\langle R\rangle$ is marked and occurs at $\langle R\rangle=2.9 \mathrm{~m}$ from the vent. From the video analyses, we can find the maximum value of $y$, which is $H$. For the white ping pong balls, the mean of all measured maximum heights is $\langle H\rangle=8.4 \mathrm{~m}$, for the small bouncy balls, this value is $\langle H\rangle=14.0 \mathrm{~m}$, for the foam

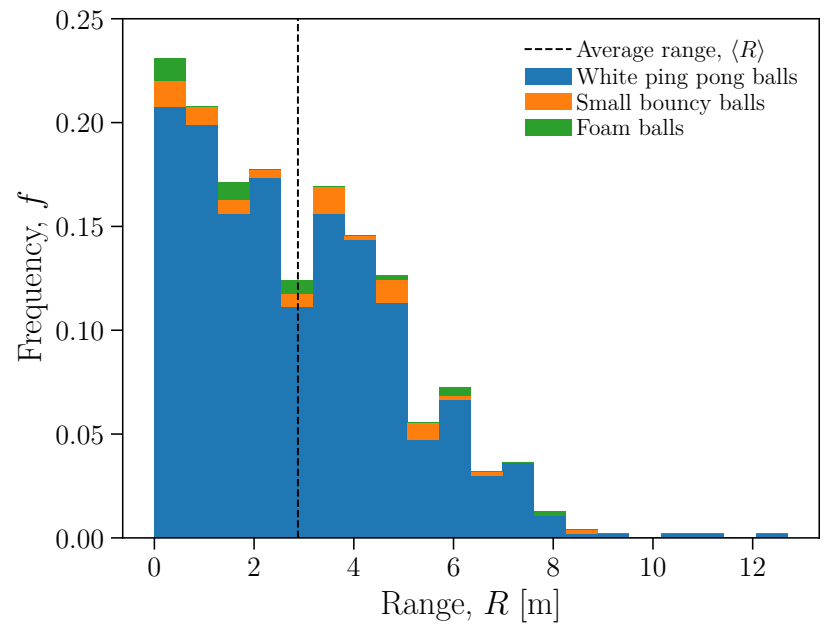

Figure 6: The frequency distribution of $R$ for each projectile type, with an arithmetic mean range $\langle R\rangle=2.9 \mathrm{~m}$. The soft football is not plotted as there was only a single projectile, however, its range was $R=4.1 \mathrm{~m}$.

balls, this value is $\langle H\rangle=15.0 \mathrm{~m}$ and for the soft football, this was $\langle H\rangle=18.4 \mathrm{~m}$. We can use Equation 17, with the fitted values of $K_{2}$ to predict $H / R$, which provides a useful test. We find that $H / R$ should vary from 2.7 to 5.8 for our projectiles with the fitted inputs from Table 2 , which, to a first-order corresponds with $\langle H\rangle /\langle R\rangle$ of $2.9,4.5,6.0$, and 4.5 , for the ping pong balls, the small bouncy balls, the foam tennis balls, and the mini football/basketball, respectively. 
Table 2 - Observed or fit parameters from data analysis.

\begin{tabular}{ccccccc}
\hline Parameter & Unit & Ping pong balls & Small bouncy balls & Foam tennis balls & Mini football & Average \\
\hline$\langle R\rangle$ & $\mathrm{m}$ & 2.9 & 3.1 & & & \\
$\langle H\rangle$ & $\mathrm{m}$ & 8.4 & 14.0 & 15.0 & 4.1 & 3.2 \\
\hline Full solution & & & & & Global fit values \\
$v_{0}$ & $\mathrm{~m} \mathrm{~s}^{-1}$ & $39.7 \pm 2.6$ & $45.0 \pm 3.8$ & $28.5 \pm 1.7$ & $28.4 \pm 1.7$ & $31.6 \pm 1.2$ \\
$K_{1}$ & - & $0.052 \pm 0.010$ & $0.072 \pm 0.017$ & $0.280 \pm 0.068$ & $0.385 \pm 0.097$ & $0.136 \pm 0.016$ \\
$v_{0} \sin (\theta)$ & $\mathrm{m} \mathrm{s}^{-1}$ & $38.8 \pm 2.5$ & $44.9 \pm 3.8$ & $28.3 \pm 1.7$ & $28.3 \pm 1.6$ & $31.2 \pm 1.1$ \\
\hline Approximation & & & & & & \\
$v_{0}$ & $\mathrm{~m} \mathrm{~s}^{-1}$ & $27.3 \pm 0.9$ & $27.3 \pm 0.9$ & $24.9 \pm 0.9$ & $24.7 \pm 0.6$ & $24.0 \pm 0.5$ \\
$K_{2}$ & - & $0.270 \pm 0.027$ & $0.560 \pm 0.070$ & $0.767 \pm 0.130$ & $1.060 \pm 0.160$ & $0.561 \pm 0.010$ \\
$v_{0} \sin (\theta)$ & $\mathrm{m} \mathrm{s}^{-1}$ & $26.7 \pm 0.8$ & $27.2 \pm 0.9$ & $24.8 \pm 0.9$ & $24.6 \pm 0.6$ & $23.7 \pm 0.4$ \\
\hline
\end{tabular}

\subsection{The limitations of observing in 2-dimensions only}

There are many unknown parameters in our set up. One of the most striking is the use of 2-dimensional video footage to track a phenomenon that is clearly occurring in 3-dimensions. This is a problem not unknown to volcanologists who often record footage of explosive phenomena from a single location and use that footage to track projectiles [Clarke et al. 2002; Gaudin et al. 2014; Taddeucci et al. 2017]. There are notable exceptions where 3-dimensional videography techniques have been deployed [Gaudin et al. 2015]. When working with the limit of 2-dimensions, as might be typical of classroom scenarios, we must explore how uncertain our predictions can be.

We consider we are an observer standing at a distance $D$ from the explosion point-source. If we imagine this scene from above, the projectile is emitted from the point-source along a trajectory that is at an angle $\alpha$ from the direct line between observer and explosion (Figure 7A). The true value of $x$, the horizontal distance of the projectile from the explosion, corresponds to an apparent distance $x^{*}$ on the apparent $x-y$ plane. The apparent $x-y$ plane is the 2-dimensional reference plane that contains the scale used by the observer to measure horizontal distances. Assuming this plane is the same as the plane in which the explosion occurs, then this geometry is given by Figure 7A (top-down view).

The ratio $x^{*} / x$ is therefore a measure of how wrong our values of $x^{*}$ are compared with the true value $x$. Using trigonometry, we can solve for $x^{*} / x$ as a function of $\alpha, x$ and $D$

$$
\frac{x^{*}}{x}=\frac{\sin (\alpha)}{1-x \cos (\alpha) / D},
$$

which is plotted in Figure 7. Here we can see that the angle $\pi / 2$ is optimal for minimizing the error on $x$. Conversely, an angle of 0 or $\pi$ render the problem illposed as there is no longer any apparent $x^{*}$. Figure 7B shows that as a general rule, if the observer is more than approximately a factor of 10 of the total range of a pro- jectile away from the explosion $(x / D<0.1)$, the result is only slightly dependent on $D$ and dominantly dependent on $\alpha$. A proper quantification of this for a given dataset of projectiles, would require more information than we can gather using 2-dimensional observations. Nonetheless, the visualization of the limitations of this problem is useful for understanding the magnitude of the uncertainties in $x$ and confirms our concerns about our uncertainties in our data presented (Figure 5).

\subsection{Extracting source parameters from an observed explosion}

It is a key goal of volcanologists to understand how energetic explosive volcanism can be, because this is directly related to the energy stored in the magma immediately prior to explosive events. In turn, that energy stored is related to the overpressure state of the magma. Output values from this experiment include $v_{0}$ (see Table 2). These values can be converted into kinetic energy $E_{k}$ at the point of explosion-termed $E_{k, 0}$-assuming $E_{k, 0}=m v_{0}^{2} / 2$. From Table 2 we can use the average $v_{0}$ and $m$ (Figure 4) for each particle type to find average values of $E_{k, 0}$, which we term $\left\langle E_{k, 0}\right\rangle$. Then, making broad assumptions that all of the population of particles of each type are accelerated to $v_{0}$ with energy $\left\langle E_{k, 0}\right\rangle$, then the total kinetic energy recorded by the particles of each type is

$$
E_{k}=\sum_{i}^{N}\left\langle E_{k, 0}\right\rangle i
$$

where there are $N$ particles of type $i$. Then the sum of all $E_{k}$ for each class of particle is the total kinetic energy.

The total value of $E_{k}$ must be equal to or less than the total energy produced by the explosion $E$. Energy is clearly lost in the form of (1) the sound wave(s) producing the audible "bang"; (2) acceleration of the bucket, which flies upward from the ground; (3) momentum transferred to the air (see discussion above about the 

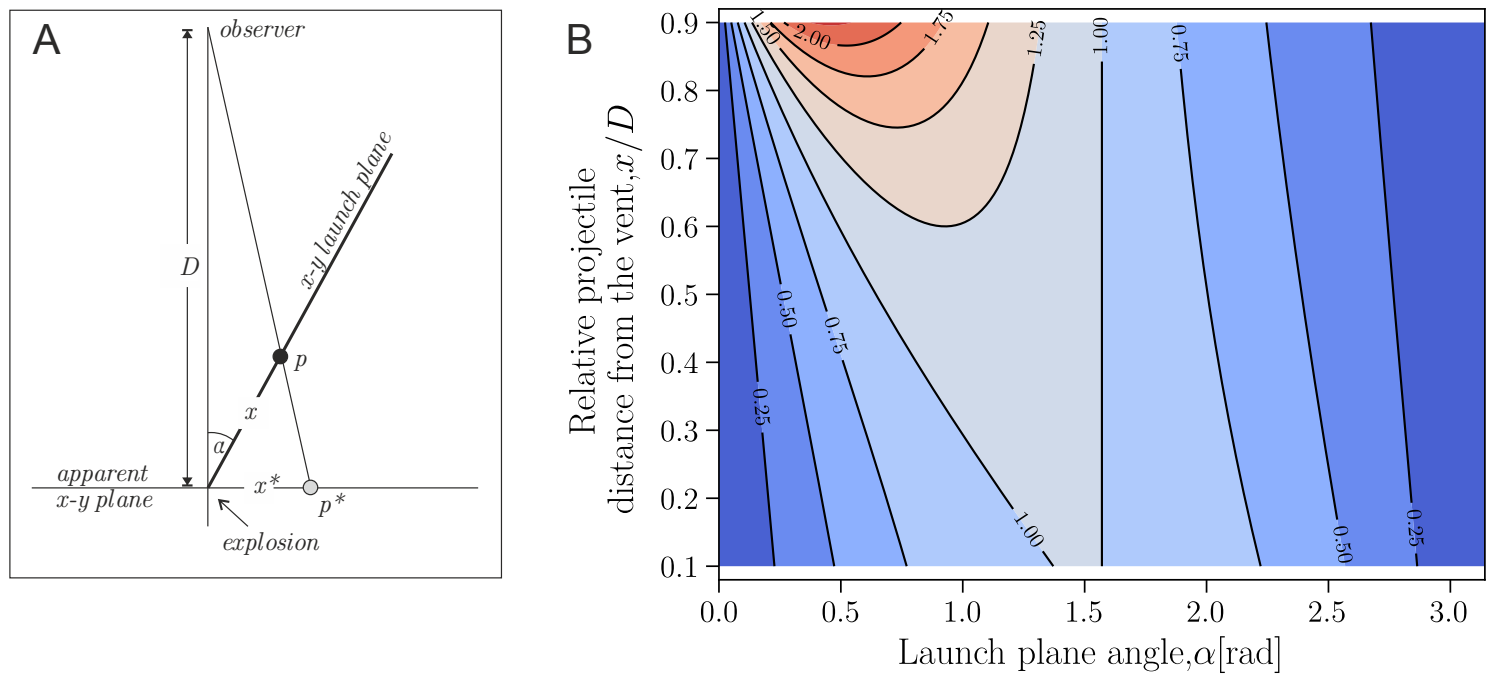

Figure 7: An assessment of the uncertainty associated with 2-dimensional tracking of a 3-dimensional explosion. [A] The geometry of the problem where the projectile position is $p$ and the apparent projectile position projected onto the apparent $x-y$ plane at $p^{*}$. The distance from the explosion and $p$ is $x$, while the distance from the explosion and $p^{*}$ is $x^{*}$. [B] The error contoured as $x^{*} / x$ in shaded colours (binned gradations at increments of $x^{*} / x$ $=0.25$ ) is a function of the relative distance of the observer from the explosion $x / D$ and the angle at which the projectile is launched $\alpha$. We note that the minimum error is $x^{*} / x=1$, which occurs for all distances when $\alpha=\pi / 2$.

air velocity); (4) the energetics of rupturing the bottle; (5) other losses such as minor amounts of heat.

We can estimate $E$ from first principles and compare with the total $E_{k}$, knowing what produces the explosion (the pressure changes associated with the phase change of nitrogen). We assume that the explosion initiates when the elastic stresses tangential to the wall of the bottle $\sigma_{\chi}$ exceed the elastic strength of the bottle in tension $\sigma_{0}$. We used a standard bottle of 1.5-litre volume made from polyethylene terephthalate. The tensile strength of this material is $\sigma_{0}=60 \mathrm{MPa}$ [Brydson 1999]. We can approximate the geometry of our bottle as a cylinder with radius $J=5 \mathrm{~cm}$, height $M=25 \mathrm{~cm}$ and wall thickness $\delta=0.8 \mathrm{~mm}$ [Snyder and Wechsler 1982]. Assuming the bottle walls do not deform, then the tangential stresses on the cylinder wall are

$$
\sigma_{\chi}=\frac{P J}{\delta},
$$

where $P$ is the pressure inside the bottle, which increases as progressively more liquid nitrogen transitions to gaseous nitrogen during heating to ambient temperature. Rupture will occur when $\sigma_{\chi}=\sigma_{0}$, therefore we can rearrange for $P$ at the time of rupture, which we call $P_{0}$ :

$$
P_{0}=\frac{\sigma_{0} \delta}{J}
$$

and the energy released at rupture can be scaled to

$$
E=P_{0} V=\pi \sigma_{0} \delta J M
$$

Using Equation 27, we find that the value of $E=\mathcal{O}\left(10^{3}\right) \mathrm{J}$ and can be dculated for our setup as $1887 \mathrm{~J}$. Equation 27 is built from the assumption that the bottle contains a pressurized ideal gas, which clearly is not the case in our experiment and ignores the phase change of nitrogen from liquid to vapour. However, if the filling fraction of liquid nitrogen is initially low, then Equation 27 is a better approximation. We make the simplifying assumption that all the energy is radiated upward to accelerate the projectiles rapidly to $v_{0}$ and neglect the fact that there are losses associated with the response of the bucket, which moves during the experiment.

In the case of the experiment conducted here, we find that $E$ derived from the sum of the particle velocities $v_{0}$ (as described) is $758 \mathrm{~J}$. This has the desirable result that it is less than the expected value of the bottle rupture energy and provides an estimate of the energetic losses not considered here. We note that in the broadest terms, the energy value calculated from the sum of particle kinetic energies is approximately half the maximum explosion energy, implying that the 'efficiency' of the explosion in terms of its ability to impart kinetic energy to the projectiles is $\sim 0.5$. This is a demonstration of how the kinetic energy of projectiles can be scaled to the source parameters of the explosion. Here, every parameter was known, but the same exercise could be conducted where we would ask, what is the approximate volume of the pressurized gas pocket that was released in the explosion? The answer to such a question would be a desirable quantity in volcanic settings as it contains information about what caused the eruption; e.g. a volcanic Taylor bubble in the case of many Strombolian explosions [Bello et al. 2012]. 


\section{Discussion}

\subsection{Comparison with other ballistic models}

A tool used commonly in volcano-monitoring efforts to predict the trajectory of ballistics is Eject! [Mastin 2001]. This computer program solves Equation 3 in combination with other governing equations that account for the thermodynamics involved in the ejection of ballistics with hot gases in eruption scenarios. Eject! also incorporates a variable drag coefficient that evolves as the Reynolds number of the projectile evolves. For these reasons, Eject! accounts for more of the possible physical processes involved in ballistic ejection at volcanic sites, compared with our solutions explored here. However, in incorporating these additional processes, the mathematical steps and overarching framework presented here is potentially obfuscated, making it a useful tool in real volcano monitoring scenarios, but less useful in trying to build a numerate pedagogic exercise.

Eject! requires input parameters from the user. These include, projectile shape, projectile density, projectile diameter, the speed of a tailwind, the initial velocity and launch angle, the temperature of the fluid (air), the distance of the landing point below the takeoff point, and some information about the rate at which the temperature drops outside the ejection zone and the size of the zone of "reduced drag". We run the software with these inputs set to be relevant to our experiment, namely, spherical projectiles of the known density and diameter (Figure 4), ambient Munich air temperature of $25^{\circ} \mathrm{C}$, the fitted velocity and launch angle presented for the global fit in Figure 5, and flat ground between take-off and landing. We assume the thermal lapse rate and the reduced drag zone are both zero. Eject! can run in either a constant drag mode, or a variable drag mode. Using constant drag mode, we find reasonable agreement between the software output and our data using $C \simeq 0.5$, which is the value predicted assuming a non-zero gas velocity for intermediate Reynolds numbers (see section 5.1; after Clift et al. [2005]). Using variable drag mode, we find excellent agreement between the software output and our data (Figure 8). This comparison demonstrates that our analysis and framework is consistent with sophisticated software designed for volcano monitoring scenarios.

\subsection{The 3-dimensional tracking of particles}

It is clear from our example analysis presented above that the use of a single camera precludes the prediction of full trajectories of projectiles. Indeed, the use of multiple cameras placed at different angles to the analogue volcanic vent would provide $3 \mathrm{D}$ information. At the lowest complexity, this would allow students to use some camera angles to estimate which projectiles really do fall in the Cartesian plane of interest for the

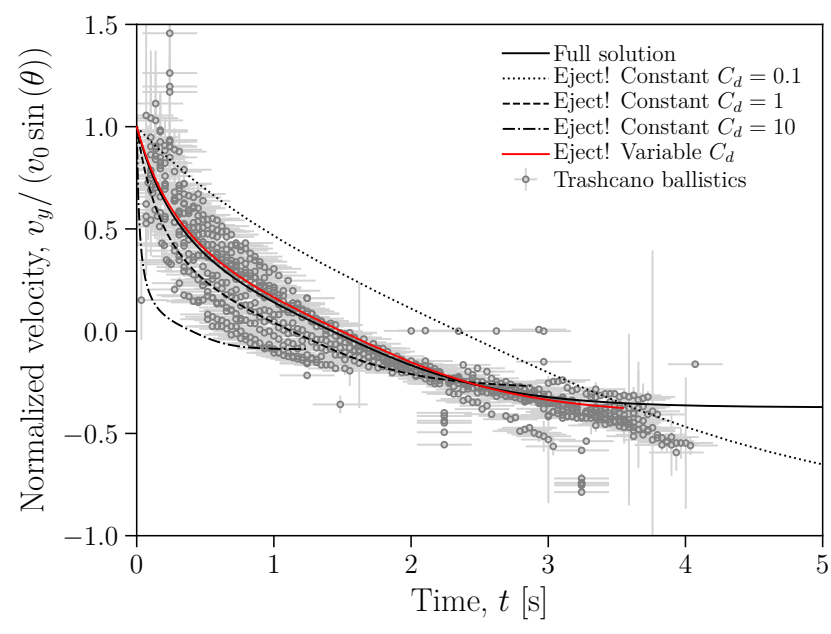

Figure 8: All data as plotted in Figure 5, but compared with output results from Eject! [Mastin 2001]. The values of $C$ (in the figure, termed $C_{d}$ after Mastin [2001]) are given for the constant- $C$ result, and in red is the full, variable- $C$ result, consistent with our full solution from Figure 5.

master camera. A more complex solution would be to use resources available online to reconstruct the full 3dimensions of the velocity vectors of the projectiles. We do not discuss exactly how to do this full 3-dimensional tracking as it is less appropriate for in-classroom deployment. Nonetheless, as an example of the efficacy of these techniques, we show the results of 3-dimensional multi-camera tracking using a trashcano experiment in which the bucket was filled with a combination of water and projectiles (Figure 9).

6.3 Working through the solutions in an undergraduate or postgraduate class

To solve the full equations, we require a numerical solution, as discussed above. Some classroom goals will be focused on data analysis and not necessarily on the details of solving ordinary differential equations. Therefore, to render this experiment of the widest utility, we provide a coded solution written for Python ${ }^{\mathrm{TM}}$, which is freely available and therefore advantageous for use in all classrooms (Listing 1). We use NumPy 1.13.0 and SciPy 1.0.0 packages here, which the user/student will need to install. The code given in Listing 1 is a tool for solving Equations 19 and 20, which are in dimensionless form, and then transforms them into dimensional form (equivalent to Equations 3 and 4 and records $v_{x}(t)$ and $v_{y}(t)$ for a given set of input parameters $g, v_{0}, K_{1}$ and $\theta$. This does not include plotting or exporting the result for use outside of the Python environment, which would need to be added. Listing 1 could be used as a tool in-class prior to the experiment to explore the range of expected behaviour and to build hypotheses about (1) the effect of drag 

A
B
C
E
$\mathrm{F}$
G
$\mathrm{H}$

Figure 9: 3-dimensional vector distributions for projectiles (red lines) and water obscuring the explosion (black points) tracked in an experiment where the bucket contained both water and particles. The individual frames in $[\mathrm{A}]-[\mathrm{H}]$ are $0.5 \mathrm{~s}$ apart and the axes are not scaled as this is for qualitative purposes only.

or particle density by altering the value of $K_{1}$, and (2) the effect of launch velocity or angle by altering those relevant inputs. Listing 1 can also be used to compare against data collected and processed to extract $v_{y}(t)$. Alternatively, the derivation of the approximate solution (Equations 7 and 8) requires basic calculus (from Equation 6 to Equations 7 and 8), making a useful exercise for practicing quantitative skills. Using Kemmer and Keller [2010], the least squares regression fit procedure, applying Equation 7 to the processed data provided as a supplementary file, can be achieved using Microsoft ${ }^{\circledR}$ Excel without the need for code-development or management skills.

\subsection{Additional considerations or discussion}

Above, we outlined a scaled example of a projectile problem where we make some implicit omissions of complexities. In this section, we acknowledge those omissions and discuss them.

We omitted other forces that affect the trajectory of a moving projectile. Many of these are demonstrably negligible in both the experimental and natural cases, such as the centrifugal or Coriolis forces due to the rotation of the Earth, or the Basset force due to the lag in boundary layer formation at the walls of accelerating projectiles in viscous fluids (see Taddeucci et al. [2017] for a review). Additionally, there is the virtual mass force $\mathbf{F}_{\mathbf{v}}$ and the pressure-gradient force $\mathbf{F}_{\mathbf{p}}$, both of which are relevant when inertia of the ambient fluid is especially important [Taddeucci et al. 2017]. de'Michieli Vitturi et al. [2010] demonstrated that pressure gradients travelling over volcanic ballistic particles can be neglected, implying that $\mathbf{F}_{\mathbf{p}} \rightarrow 0$ and Bertin [2017] found that the $\mathbf{F}_{\mathbf{v}}$ can be neglected. Therefore, we do not consider these here. Were these force components necessary, they would be additive to the righthand side of Equation 1.

The force of buoyancy $\mathbf{F}_{\mathbf{b}}$ occurs due to the density difference between the projectile and the ambient fluid and is relevant if the projectile is of sufficiently low density. It is given by $\mathbf{F}_{\mathbf{b}}=\rho V \mathbf{g}$, where $V$ is the volume of the projectile. This force is often included in the calculation because it does not depend on $\mathrm{v}$ and is simply a correction to the term g. A useful scaling to work out if $\mathbf{F}_{\mathbf{b}}$ must be considered is that $\mathbf{F}_{\mathbf{b}} / \mathbf{F}_{\mathbf{g}} \sim \rho / \rho_{p}$, where $\rho_{p}$ is the density of the particles, and values of this ratio far in excess of unity would imply that $\mathbf{F}_{\mathbf{b}}$ is non-negligble. In our case, $\rho / \rho_{p} \sim 100$ (see Figure 4 for densities $\rho_{p}$ ) and so the value of $\mathbf{F}_{\mathbf{b}}$ could be a valuable addition to the analysis by adding its contribution to the right side of Equation 1. In the volcanic scenario, $\rho_{p} \sim 10^{3} \mathrm{~kg} \mathrm{~m}^{-3}$ and so $\mathbf{F}_{\mathbf{b}} / \mathbf{F}_{\mathbf{g}} \sim 10^{-3}$ and the buoyancy force can be neglected.

The Magnus force $\mathbf{F}_{\mathbf{M}}$ is the sideways force generated by rotation of a projectile moving in a fluid, and is most commonly discussed in relation to a footballer's ability to curve the trajectory of the ball around other players by subjecting the ball to spin at the moment of launch. This force is given by

$$
\mathbf{F}_{\mathbf{M}}=\frac{1}{2} \rho C_{l} A \mathbf{v}^{2} \frac{\omega \mathbf{n v}}{|\omega \mathbf{n v}|}
$$

where $\omega$ is the angular velocity of the projectile, $C_{l}$ is the lift coefficient, and $\mathbf{n}$ is a unit vector normal to the $x-y$ plane of launch. Clearly, $\mathbf{F}_{\mathbf{M}}$ becomes nonnegligible at high $\omega$. There has been little direct analysis of how important the Magnus force could be in volcanic scenarios and it is not an easy-to-measure parameter without 3-dimensional observations of curved trajectories in either the laboratory or in nature [Bertin 2017; Taddeucci et al. 2017].

As an engineering approximation of the complex phenomenon of lift, the lift force $F_{1}$ can account for the forces that act perpendicular to the angle of attack or an object subjected to a bypassing fluid stream. The form for $\mathbf{F}_{1}$ is similar to $\mathbf{F}$ and is

$$
\mathbf{F}_{1}=\frac{1}{2} \rho C_{l} A \mathbf{v}^{2}
$$

which includes the lift coefficient $C_{l}$, found by experiment for different shaped objects. At low velocities, this 
force is usually negligible compared with drag forces $\mathbf{F}$ and in both the natural and the experimental case, this is rarely considered [c.f. Bertin 2017; Taddeucci et al. 2017].

\subsection{Volcanic projectiles}

Herein we use the scaling for the maximum range $R$ that a projectile can reach. This is often the most important first-order scaling required at volcanoes because it represents the blast-zone in which ballistic hazards are high. Once these physical concepts are taught to a class, it is a simple extension to discuss the hazards associated with typical volcanic eruptions, given a reasonable range of values for $v_{0}, \theta, A$ and $C$ [Breard et al. 2014; Fitzgerald et al. 2014]. Large eruptions with a high intensity convey a large $v_{0}$ and therefore their typical blast radius at which the maximum $\mathrm{R}$ occurs can be large [Fitzgerald et al. 2014].

Field evidence for volcanic projectiles can be found in recent eruptions and in the geological record. For example, ballistic volcanic bombs produce impact craters whose size typically scales with the size of the bomb and the speed with which it impacted the ground [Breard et al. 2014; Fitzgerald et al. 2014]. In ignimbrites, proximal facies often contain ballistic bombs that can be recognised by the sag-features of distorted surrounding bedding or by the fact that their size is inconsistent with the finer matrix [Branney et al. 2002].

Observations of the distribution of impact craters around a vent yields information about the distribution of $\theta$ from a given eruption. Such information has been used to infer that the subsurface vent is inclined with respect to the vertical [Gurioli et al. 2013; Kilgour et al. 2010].

\subsection{How effective can trashcano be as a teaching tool?}

In order to explore the efficacy of this teaching technique in classrooms and laboratory practical sessions, we provide some anecdotal evidence from laboratories in different countries around the world. We spoke to 4 staff members at different universities, all of whom have deployed variants of the trashcano model in a teaching environment. In each case, while the experiment itself was broadly similar, the details of the level, complexity, and depth to which the results were interrogated varies (see Table 1 for a range of possible complexity levels and Figure 10 for examples of the trashcano experiment performed as part of teaching programs around the world). Here we summarize each deployment of this exercise and provide some anecdotal evidence for its efficacy, leaving a quantitative approach to future work.

\section{Example 1 (A. Graettinger pers. comm.)}

Here trashcano is used for an introductory geoscience course as part of an undergraduate curricu- lum. The experiment is used as a mechanism to entice students to be active learners, and as an opportunity to practice observational, hypothesis-driven science, including data collection from large scale phenomena. The instructor reports that they typically set the students the task of measuring the height and range of the projectiles, and, as a mathematical component, of calculating the pressure that drove the eruption using the technique described in Harpp et al. [2005]. When asked if the exercise is effective as a teaching tool, this instructor reflects, "I gauge the success of the demonstrations for classroom methods through student engagement (through question asking, talking about the experiment even after it is over, and commentary on submitted assignments) and it [is] frequently one of the favorite activities in class."

\section{Example 2 (S. Schneider pers. comm.)}

In this example trashcano is used as a general outreach activity or as a part of a half- or full-day workshop on natural hazards. Interestingly, this instructor feels that the spectacle of the experiment can spark interest in volcanic phenomena in those who are not especially interested in the application of mathematics to physical problems. In their teaching practice, they have noticed that students benefit from having an active break from the classroom in what they describe as "learn stops" or periods of non-classroom learning. They highlight that the fun aspects of the experiment are essential in terms of the feedback about the experience from both the extended teaching staff involved, the students, and even the parents of younger students involved.

\section{Example 3 (B. Kennedy pers. comm.)}

Here trashcano is used as part of an undergraduate university curriculum in volcanology. The instructor has experimented with different formal tasks to accompany the experimental demonstration in order to best engage the students. Direct quotes from students and feedback forms extracted by the instructor reflect the enthusiasm that can be engendered with this experiment.

\section{Example 4 (J. Barclay pers. comm.)}

This instructor has used trashcano at a wide range of levels, from demonstrating some physical principles (such as changes from liquid to a gas and the resultant implications for pressure and volume) to students in primary education (approximately in the age range 6-11), to a more complex level (secondary education), as a mechanism to visually convey the concepts of momentum transfer as the projectiles initially collide with one another before being finally ejected on ballistic trajectories. At the university undergraduate level, this 

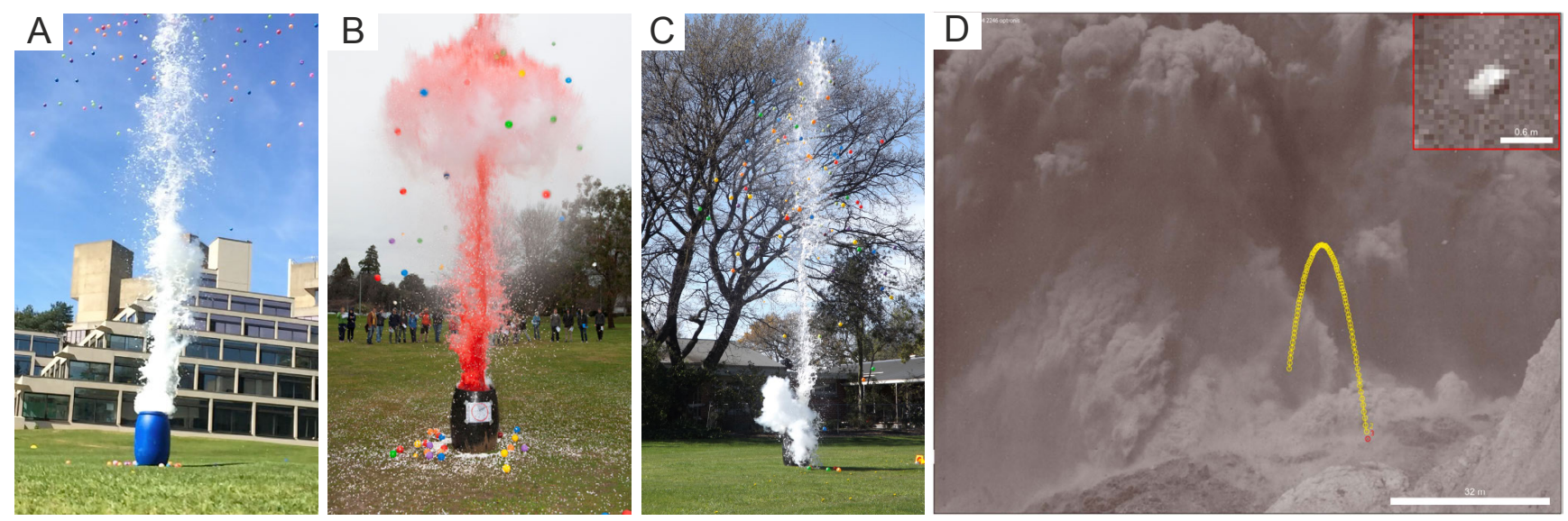

Figure 10: Trashcano is carried out in laboratories around the world as a teaching tool and demonstration of aspects of volcanic eruption dynamics. Panels [A]-[C] show examples of trashcano in which water is used in the bucket along with the particles, with example [A] from the University of East Anglia in the U.K., courtesy of Prof. Jenni Barclay, and examples [B] and [C] from the University of Canterbury in Christchurch, New Zealand. In panel [D] we give an example of a volcanic ballistic projectile (red marker) tracked through time in frames prior to the frame presented (yellow points) during explosive volcanism at Batu Tara volcano, Indonesia (see Taddeucci et al. [2017] for details). The image in [D] is reproduced from Taddeucci et al. [2017] with permission of the American Geophysical Union.

instructor deploys trashcano as a way to demonstrate the concepts involved in Vulcanian volcanic eruptions, ideas of clast size distribution in deposits from eruptions, eruption size or magnitude, or hazards from ballistics around volcanoes. This demonstrates the diversity of possible applications of the experiment.

None of the instructors we spoke to reported the use of trashcano as a tool for developing predictions of ballistic trajectories, nor the use of calculus associated with the trashcano experiment. We propose, therefore, that our framework can complement existing uses of the experiment, adding mathematical value to a fun demonstration, and thereby going some way to improving "quantitative literacy" in the geosciences [Manduca et al. 2008; Wenner et al. 2009; Wenner et al. 2011].

\section{Conclusions}

We give a quantitative framework-and example code for Python ${ }^{\mathrm{TM}}$ - for solving ballistic trajectory problems in 2-dimensional Cartesian coordinates. We discuss the problem of dealing with a 2-dimensional plane in what is actually a 3-dimensional problem and provide a method for assessing the discrepancies associated with this limitation. For classes aimed at undergraduate level, we propose that the full framework developed is useful along with previously developed computer programs such as Eject! [Mastin 2001]. However, for classes aimed at different levels of complexity, we provide limiting simpler solutions that recover the basic behaviour of the full solution. We show how this problem can be related to volcanic phenomena and how source parameters can be calculated from this inclass experiment, similar to the way in which they are computed for volcanic phenomena. Useful future work would include providing open-source and easy-to-use computational tools to track ballistic trajectories in 3dimensions using multiple cameras or drones.

\section{Acknowledgements}

This article was written as a result of the Physics of Volcanoes (formally: Vulkanologie II) course built and taught by Fabian Wadsworth and Bettina Scheu 20162017, which was supported by the Studi_forscht@GEO program of Ludwig-Maximilians-Universität (grant numbers W16_F18 and W16_F6). Holly Unwin was supported by the Summer Internship Programme of the University of Oxford partnered with LudwigMaximilians-Universität, Munich. We thank the staff at the Pinakothek der Moderne in Munich for their forbearance during the explosion experiments conducted outside their building and the Polizei München for not making a fuss. Inventive variations of this experiment have been conducted in volcanology classes worldwide and we acknowledge the large input from many of these labs via helpful online materials provided open-source and free of charge (in particular the Oregon State University page: http://volcano.oregonstate.edu/depthcharge-eruption-column). A particularly informative web-based resource for classical mechanics was developed by Prof. Richard Fitzpatrick (University of Texas). We are grateful to Alexa Van Eaton, Alison Graettinger, Jenni Barclay, and Simon Schneider for sharing their experiences of trashcano in their respective classrooms 
Listing 1 - Example code solution to the full model given in Equations 3 and 4 written for Python ${ }^{\mathrm{TM}}$.

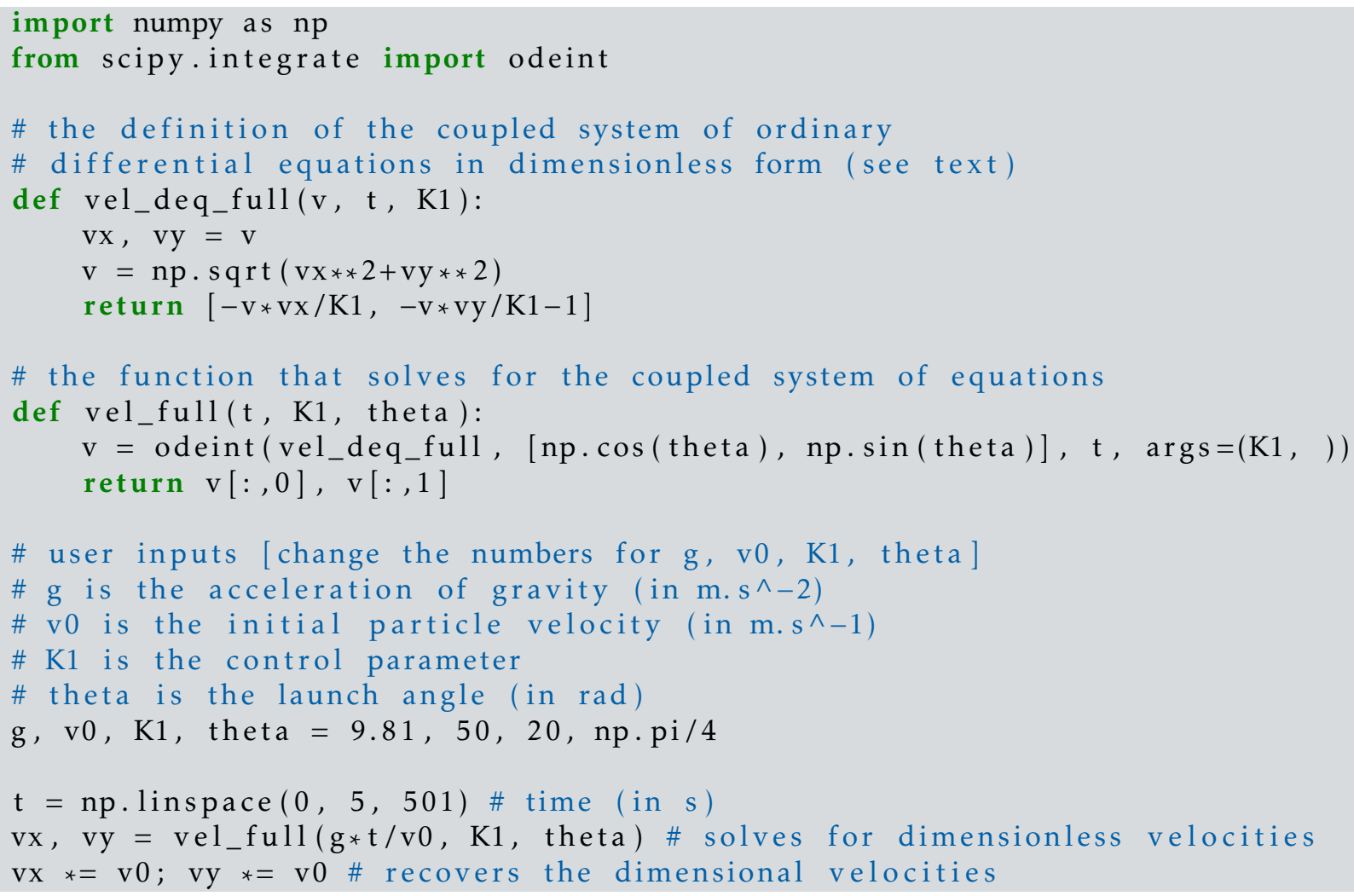

and events. Finally, we thank Thomas Kunzmann for help during the experiment, and Ed Llewellin for useful discussion about trigonometry and pedagogical exercises in general and our reviewers and editor, Mike James, for constructive feedback that has led to significant improvements.

\section{Author CONTRibutions}

Fabian Wadsworth and Bettina Scheu conceptualized the study. Fabian Wadsworth, Jérémie Vasseur, Gilles Seropian and Holly Unwin led the data analysis. Holly Unwin, Julia Holzmueller, Bettina Scheu, Taylor Witcher, Janina Adolf, Francisco Cáceres, Ana Casas, Valeria Cigala, Mathieu Colombier, Shane Cronin, Marcel Cronin, Leticia Freitas Guimarães, Laura Höltgen, Sönke Stern, Caron Vossen and Natalie Weichselgartner all collected the data. Adrian Teissier and Ben Kennedy performed the 3D data analysis. Alexandra Clement provided pedagogical context. Fabian Wadsworth, Shane Cronin, Ulrich Kueppers, Ben Kennedy, Bettina Scheu, and Donald Dingwell led the writing of the manuscript, with contributions from all authors.

\section{Data aVailability}

The $v_{y}(t)$ data used in Figure 5 are provided as a supplementary dataset for the use in classroom exercises Presses universitaires de strasbourg where the full experiment cannot be deployed.

\section{COPYRight NOTICE}

(C) The Author(s) 2018. This article is distributed under the terms of the Creative Commons Attribution 4.0 International License, which permits unrestricted use, distribution, and reproduction in any medium, provided you give appropriate credit to the original author(s) and the source, provide a link to the Creative Commons license, and indicate if changes were made.

\section{REFERENCES}

Beichner, R. J., J. M. Saul, D. S. Abbott, J. J. Morse, D. Deardorff, R. J. Allain, S. W. Bonham, M. H. Dancy, and J. S. Risley (2007). "The student-centered activities for large enrollment undergraduate programs (SCALE-UP) project". Research-based reform of university physics 1.1 , pp. 2-39.

Bello, E. D., E. W. Llewellin, J. Taddeucci, P. Scarlato, and S. J. Lane (2012). "An analytical model for gas overpressure in slug-driven explosions: Insights into Strombolian volcanic eruptions". Journal of Geophysical Research: Solid Earth 117.B2, pp. 1-18. DoI: 10 . $1029 / 2011$ jb008747. 
Bertin, D. (2017). "3-D ballistic transport of ellipsoidal volcanic projectiles considering horizontal wind field and variable shape-dependent drag coefficients". Journal of Geophysical Research: Solid Earth 122.2, pp. 1126-1151. Dor: 10. 1002/2016 jb013320.

Blackburn, E. A., L. Wilson, and R. S. J. Sparks (1976). "Mechanisms and dynamics of strombolian activity". Journal of the Geological Society 132.4, pp. 429-440. DoI: 10.1144 /gs jgs.132.4.0429.

Branney, M. J., P. Kokelaar, and B. P. Kokelaar (2002). "Pyroclastic density currents and the sedimentation of ignimbrites". Geological Society of London.

Breard, E., G. Lube, S. Cronin, R. Fitzgerald, B. Kennedy, B. Scheu, C. Montanaro, J. White, M. Tost, J. Procter, and A. Moebis (2014). "Using the spatial distribution and lithology of ballistic blocks to interpret eruption sequence and dynamics: August 62012 Upper Te Maari eruption, New Zealand". Journal of Volcanology and Geothermal Research 286, pp. 373386. Dor: 10.1016/ j . jvolgeores.2014.03.006.

Brydson, J. A. (1999). Plastics materials. Elsevier.

Chouet, B., N. Hamisevicz, and T. R. McGetchin (1974). "Photoballistics of volcanic jet activity at Stromboli, Italy". Journal of Geophysical Research 79.32, pp. 4961-4976. DoI: 10.1029/ jb079i032p04961.

Clarke, A., A. Neri, B. Voight, G. Macedonio, and T. Druitt (2002). "Computational modelling of the transient dynamics of the August 1997 Vulcanian explosions at Soufriere Hills Volcano, Montserrat: influence of initial conduit conditions on near-vent pyroclastic dispersal". Geological Society of London Memoirs 21, pp. 319-348. DoI: 10. 1144 / GSL . MEM . 2002. 021.01 .15$.

Clift, R., J. R. Grace, and M. E. Weber (2005). Bubbles, drops, and particles. Dover Publications Inc.

De’Michieli Vitturi, M., A. Neri, T. E. Ongaro, S. L. Savio, and E. Boschi (2010). "Lagrangian modeling of large volcanic particles: Application to Vulcanian explosions". Journal of Geophysical Research 115.B8. DOI: $10.1029 / 2009$ jb007111.

Deslauriers, L., E. Schelew, and C. Wieman (2011). "Improved Learning in a Large-Enrollment Physics Class". Science 332.6031, pp. 862-864. Dor: 10.1126/ science. 1201783.

Dohaney, J., E. Brogt, B. Kennedy, T. M. Wilson, and J. M. Lindsay (2015). "Training in crisis communication and volcanic eruption forecasting: design and evaluation of an authentic role-play simulation". Journal of Applied Volcanology 4.1. DoI: 10 . 1186 / s13617-015-0030-1.

Fagents, S. A. and L. Wilson (1993). "Explosive volcanic eruptions-VII. The ranges of pyroclasts ejected in transient volcanic explosions". Geophysical Journal International 113.2, pp. 359-370. Dor: $10.1111 / \mathrm{j}$. 1365-246x. 1993. tb00892.x.

Fitzgerald, R., K. Tsunematsu, B. Kennedy, E. Breard, G. Lube, T. Wilson, A. Jolly, J. Pawson, M. Rosenberg, and S. Cronin (2014). "The application of a calibrated 3D ballistic trajectory model to ballistic hazard assessments at Upper Te Maari, Tongariro". Journal of Volcanology and Geothermal Research 286, pp. 248262. Dor: 10.1016/ j . jvolgeores.2014.04.006.

Freeman, S., S. L. Eddy, M. McDonough, M. K. Smith, N. Okoroafor, H. Jordt, and M. P. Wenderoth (2014). "Active learning increases student performance in science, engineering, and mathematics". Proceedings of the National Academy of Sciences 111.23, pp. 84108415. Dor: $10.1073 /$ pnas. 1319030111.

Froyd, J. E. (2007). "Evidence for the efficacy of student-active learning pedagogies". Project Kaleidoscope 66.1, pp. 64-74.

- (2008). "White paper on promising practices in undergraduate STEM education". Commissioned paper for the Evidence on Promising Practices in Undergraduate Science, Technology, Engineering, and Mathematics (STEM) Education Project, The National Academies Board on Science Education.

Gaudin, D., J. Taddeucci, P. Scarlato, E. Del Bello, B. Houghton, T. Orr, D. Andronico, and U. Kueppers (2015). "Game of thrown bombs in 3D: using high speed cameras and photogrammetry techniques to reconstruct bomb trajectories at Stromboli (Italy)". AGU Fall Meeting Abstracts. V51F-3087.

Gaudin, D., M. Moroni, J. Taddeucci, P. Scarlato, and L. Shindler (2014). "Pyroclast Tracking Velocimetry: A particle tracking velocimetry-based tool for the study of Strombolian explosive eruptions". Journal of Geophysical Research: Solid Earth 119.7, pp. 5369-5383. DoI: 10 . 1002 / 2014 jb011095.

Gurioli, L., A. Harris, L. Colò, J. Bernard, M. Favalli, M. Ripepe, and D. Andronico (2013). "Classification, landing distribution, and associated flight parameters for a bomb field emplaced during a single major explosion at Stromboli, Italy". Geology 41.5, pp. 559562. DoI: $10.1130 / \mathrm{g} 33967$. 1.

Haak, D. C., J. HilleRisLambers, E. Pitre, and S. Freeman (2011). "Increased Structure and Active Learning Reduce the Achievement Gap in Introductory Biology". Science 332.6034, pp. 1213-1216. Dor: 10 . 1126 / science. 1204820.

Hake, R. R. (1998). "Interactive-engagement versus traditional methods: A six-thousand-student survey of mechanics test data for introductory physics courses". American Journal of Physics 66.1, pp. 64-74. DoI: $10.1119 / 1.18809$.

Harpp, K. S., A. M. Koleszar, and D. J. Geist (2005). "Volcanoes in the Classroom: A Simulation of an Eruption Column". Journal of Geoscience Education 53.2, pp. 173-175. Dor: 10.5408/1089-9995-53.2. 173.

Harpp, K. S. and W. J. Sweeney (2002). "Simulating a Volcanic Crisis in the Classroom". Journal of Geoscience Education 50.4, pp. 410-418. DoI: $10.5408 /$ 1089-9995-50.4 .410. 
Kemmer, G. and S. Keller (2010). "Nonlinear leastsquares data fitting in Excel spreadsheets". Nature Protocols 5.2, pp. 267-281. DoI: 10 . 1038 / nprot . 2009. 182.

Kilgour, G., V. Manville, F. D. Pasqua, A. Graettinger, K. Hodgson, and G. Jolly (2010). "The 25 September 2007 eruption of Mount Ruapehu, New Zealand: Directed ballistics, surtseyan jets, and ice-slurry lahars". Journal of Volcanology and Geothermal Research 191.1-2, pp. 1-14. Dor: 10 . 1016 / j . jvolgeores . 2009. 10.015.

Knight, J. K. and W. B. Wood (2005). “Teaching More by Lecturing Less". Cell Biology Education 4.4, pp. 298310. DoI: 10.1187/05-06-0082.

Linde, A. T., S. Sacks, D. Hidayat, B. Voight, A. Clarke, D. Elsworth, G. Mattioli, P. Malin, E. Shalev, S. Sparks, and C. Widiwijayanti (2010). "Vulcanian explosion at Soufrière Hills Volcano, Montserrat on March 2004 as revealed by strain data". Geophysical Research Letters 37.19, n/a-n/a. Dor: 10 . 1029 / 2009 gl041988.

Loudin, M. (2004). "Where in the World Will We Find Our Future Geoscientists?: One Employer's Perspective”. AGU Fall Meeting Abstracts.

Lube, G., E. C. Breard, S. J. Cronin, J. N. Procter, M. Brenna, A. Moebis, N. Pardo, R. B. Stewart, A. Jolly, and N. Fournier (2014). "Dynamics of surges generated by hydrothermal blasts during the $6 \mathrm{Au}-$ gust 2012 Te Maari eruption, Mt. Tongariro, New Zealand". Journal of Volcanology and Geothermal Research 286, pp. 348-366. DoI: 10 . 1016 / j . jvolgeores.2014.05.010.

Macdonald, R. H., L. Srogi, and G. B. Stracher (2000). "Building the Quantitative Skills of Students in Geoscience Courses". Journal of Geoscience Education 48.4, pp. 409-412. Dor: 10 . 5408 / 1089 - $9995-48$. 4.409.

Manduca, C. A., E. Baer, G. Hancock, R. H. Macdonald, S. Patterson, M. Savina, and J. Wenner (2008). "Making Undergraduate Geoscience Quantitative". Eos, Transactions American Geophysical Union 89.16, p. 149. Dor: 10.1029/2008eo 160001.

Martin, B. S. (1993). "Interactive Modelling of Open Magma Systems with Spreadsheets". Journal of Geological Education 41.2, pp. 164-169. DOI: 10.5408 / 0022-1368-41.2.164.

Mastin, L. G. (2001). "A simple calculator of ballistic trajectories for blocks ejected during volcanic eruptions". US Geological Survey 2001-45. Dor: 10.3133/ of 0145 .

Meijering, E., O. Dzyubachyk, and I. Smal (2012). "Methods for Cell and Particle Tracking". Imaging and Spectroscopic Analysis of Living Cells - Optical and Spectroscopic Techniques. Elsevier, pp. 183-200. DoI: 10. 1016/b978-0-12-391857-4.00009-4.
Mueller, S., B. Scheu, O. Spieler, and D. B. Dingwell (2008). "Permeability control on magma fragmentation". Geology 36.5, p. 399. Dor: 10.1130/g24605a. 1.

Penner, D. E., R. Lehrer, and L. Schauble (1998). "From Physical Models to Biomechanics: A Design-Based Modeling Approach". Journal of the Learning Sciences 7.3-4, pp. 429-449. Dor: 10 . $1080 / 10508406$. 1998 . 9672060.

Prince, M. (2004). "Does Active Learning Work? A Review of the Research". Journal of Engineering Education 93.3, pp. 223-231. Dor: 10 . 1002 / j . 2168 9830.2004. tb00809.x.

Rust, A., K. Cashman, and H. Wright (2008). "Fudge Factors in Lessons on Crystallization, Rheology and Morphology of Basalt Lava Flows". Journal of Geoscience Education 56.1, pp. 73-80. DoI: 10.5408 / 1089-9995-56.1.73.

Schneider, C. A., W. S. Rasband, and K. W. Eliceiri (2012). "NIH Image to ImageJ: 25 years of image analysis". Nature Methods 9.7, pp. 671-675. Dor: 10 . 1038 /nmeth . 2089.

Sigurdsson, H., B. Houghton, S. McNutt, H. Rymer, and J. Stix (2015). The encyclopedia of volcanoes. 2nd ed. Elsevier.

Snyder, J. A. and R. L. Wechsler (1982). Plastic bottle. US Patent 4,318,489.

Taddeucci, J., M. A. Alatorre-Ibargüengoitia, O. CruzVázquez, E. D. Bello, P. Scarlato, and T. Ricci (2017). "In-flight dynamics of volcanic ballistic projectiles". Reviews of Geophysics 55.3, pp. 675-718. DOI: 10 . 1002 / 2017 rg000564.

Taylor, G. (1950). "The Formation of a Blast Wave by a Very Intense Explosion. II. The Atomic Explosion of 1945". Proceedings of the Royal Society A: Mathematical, Physical and Engineering Sciences 201.1065, pp. 175-186. DoI: 10.1098/rspa. 1950.0050.

Thalin, G. (2013). Deshaker. URL: http : / / www . guthspot . se / video / deshaker . htm (visited on 09/15/2018).

Tsunematsu, K., Y. Ishimine, T. Kaneko, M. Yoshimoto, T. Fujii, and K. Yamaoka (2016). "Estimation of ballistic block landing energy during 2014 Mount Ontake eruption". Earth, Planets and Space 68.1. Dor: 10.1186/s40623-016-0463-8.

Wenner, J. M., H. E. Burn, and E. M. Baer (2011). “The math you need, when you need it: Online modules that remediate mathematical skills in introductory geoscience courses". Journal of College Science Teaching 41.1, p. 16.

Wenner, J., E. Baer, C. Manduca, R. H. Macdonald, S. Patterson, and M. Savina (2009). "The Case for Infusing Quantitative Literacy into Introductory Geoscience Courses". Numeracy 2.1. Dor: 10.5038/19364660.2.1.4.

Wirth, K. R. (2003). "Using an M\&M ${ }^{\circledR}$ magma chamber to illustrate magmatic differentiation". 2003 Seattle Annual Meeting. 120-18. 